\title{
THE CHANDRA COSMOS SURVEY. III. OPTICAL AND INFRARED IDENTIFICATION OF X-RAY POINT SOURCES
}

\author{
F. Civano ${ }^{1}$, M. Elvis ${ }^{1}$, M. Brusa ${ }^{2}$, A. Comastri ${ }^{3}$, M. Salvato ${ }^{2,4}$, G. Zamorani $^{3}$, T. Alderoft ${ }^{1}$, A. Bongiorno $^{2,5}$, P. Capak $^{6}$, \\ N. Cappellutis ${ }^{3}$, M. Cisternas ${ }^{7}$, F. Fiore ${ }^{5}$, A. Fruscione ${ }^{1}$, H. HaO ${ }^{1}$, J. Kartaltepe ${ }^{8}$, A. Koekemoer ${ }^{9}, 10$, R. Gilli ${ }^{3}$,

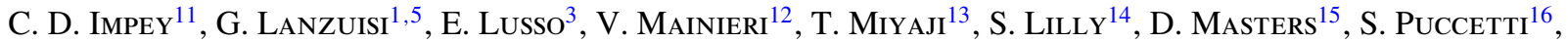 \\ K. Schawinski ${ }^{17}$, N. Z. Scoville ${ }^{6}$, J. Silverman ${ }^{18}$, J. Trump ${ }^{11}$, M. UrRY ${ }^{17}$, C. Vignali ${ }^{3}, 19$, and N. J. Wright ${ }^{1}$ \\ ${ }^{1}$ Harvard Smithsonian Center for Astrophysics, 60 Garden Street, Cambridge, MA 02138, USA \\ ${ }^{2}$ Max Planck Institut für Extraterrestrische Physik, Giessenbach Strasse 1, D-85748 Garching, Germany \\ ${ }^{3}$ INAF-Osservatorio Astronomico di Bologna, via Ranzani 1, I-40127 Bologna, Italy \\ ${ }^{4}$ Excellence Cluster, Boltzmannstrass 2, Garching D-85748, Germany \\ ${ }^{5}$ INAF-Osservatorio Astronomico di Roma, via Frascati 33, Monteporzio-Catone (Roma) I-00040, Italy \\ ${ }^{6}$ California Institute of Technology, 1201 East California Boulevard, Pasadena, CA 91125, USA \\ ${ }^{7}$ Max-Planck-Institut für Astronomie, Konigstuhl 17, D-69117 Heidelberg, Germany \\ ${ }^{8}$ Institute for Astronomy, 2680 Woodlawn Drive, University of Hawaii, Honolulu, HI 96822, USA \\ ${ }^{9}$ Space Telescope Science Institute, 3700 San Martin Drive, Baltimore, MD 21218, USA \\ ${ }^{10}$ Department of Physics and Astronomy, University of California, 900 University Avenue, Riverside, CA 92521, USA \\ ${ }^{11}$ Steward Observatory, University of Arizona, 933 North Cherry Avenue, Tucson, AZ 85721, USA \\ ${ }^{12}$ ESO, Karl-Schwarzschild-Strasse 2, D-85748 Garching, Germany \\ ${ }^{13}$ Universidad Nacional Autonoma de Mexico-Ensenada, Km. 103 Carretera Tijuana-Ensenada, 22860 Ensenada, Mexico \\ ${ }^{14}$ Institute of Astronomy, Swiss Federal Institute of Technology, 8093 Zurich, Switzerland \\ ${ }^{15}$ Observatories of the Carnegie Institution of Washington, Pasadena, CA 91101, USA \\ ${ }^{16}$ ASI Science Data Center, via Galileo Galilei, I-00044 Frascati, Italy \\ ${ }^{17}$ Department of Physics, Yale University, New Haven, CT 06511, USA \\ 18 Institute for the Physics and Mathematics of the Universe (IPMU), University of Tokyo, Kashiwanoha 5-1-5, Kashiwa, Chiba 277-8568, Japan \\ ${ }_{19}$ Dipartimento di Astronomia, Universitá di Bologna, via Ranzani 1, I-40127 Bologna, Italy \\ Received 2011 November 18; accepted 2012 May 21; published 2012 August 2
}

\begin{abstract}
The Chandra COSMOS Survey (C-COSMOS) is a large, $1.8 \mathrm{Ms}$, Chandra program that has imaged the central $0.9 \mathrm{deg}^{2}$ of the COSMOS field down to limiting depths of $1.9 \times 10^{-16} \mathrm{erg} \mathrm{cm}^{-2} \mathrm{~s}^{-1}$ in the soft $(0.5-2 \mathrm{keV})$ band, $7.3 \times 10^{-16} \mathrm{erg} \mathrm{cm}^{-2} \mathrm{~s}^{-1}$ in the hard (2-10 keV) band, and $5.7 \times 10^{-16} \mathrm{erg} \mathrm{cm}^{-2} \mathrm{~s}^{-1}$ in the full $(0.5-10 \mathrm{keV})$ band. In this paper we report the $i, K$, and $3.6 \mu \mathrm{m}$ identifications of the $1761 \mathrm{X}$-ray point sources. We use the likelihood ratio technique to derive the association of optical/infrared counterparts for $97 \%$ of the X-ray sources. For most of the remaining $3 \%$, the presence of multiple counterparts or the faintness of the possible counterpart prevented a unique association. For only $10 \mathrm{X}$-ray sources we were not able to associate a counterpart, mostly due to the presence of a very bright field source close by. Only two sources are truly empty fields. The full catalog, including spectroscopic and photometric redshifts and classification described here in detail, is available online. Making use of the large number of X-ray sources, we update the "classic locus" of active galactic nuclei (AGNs) defined 20 years ago in soft X-ray surveys and define a new locus containing $90 \%$ of the AGNs in the survey with full-band luminosity $>10^{42} \mathrm{erg} \mathrm{s}^{-1}$. We present the linear fit between the total $i$-band magnitude and the X-ray flux in the soft and hard bands, drawn over two orders of magnitude in X-ray flux, obtained using the combined C-COSMOS and XMM-COSMOS samples. We focus on the X-ray to optical flux ratio $(\mathrm{X} / \mathrm{O})$ and we test its known correlation with redshift and luminosity, and a recently introduced anti-correlation with the concentration index $(C)$. We find a strong anti-correlation (though the dispersion is of the order of $0.5 \mathrm{dex}$ ) between $\mathrm{X} / \mathrm{O}$ computed in the hard band and $C$ and that $90 \%$ of the obscured AGNs in the sample with morphological information live in galaxies with regular morphology (bulgy and disky/spiral), suggesting that secular processes govern a significant fraction of the black hole growth at X-ray luminosities of $10^{43}-10^{44.5} \mathrm{erg} \mathrm{s}^{-1}$. We also investigate the degree of obscuration of the sample using the hardness ratio, and we compare the X-ray color with the near-infrared to optical color.
\end{abstract}

Key words: galaxies: active - surveys - X-rays: galaxies: clusters

Online-only material: color figures, supplemental data (FITS) file (tar.gz)

\section{INTRODUCTION}

There is now much evidence showing that galaxies and their central supermassive black holes (SMBHs) undergo closely coupled evolution. The masses of SMBHs in the nuclei of most nearby bulges are tightly proportional to bulge luminosity and velocity dispersion (Magorrian et al. 1998; Gebhardt et al. 2000; Ferrarese \& Merritt 2000; Merloni et al. 2010). Most SMBH growth occurs during their active phases (the "Soltan argument,"
Soltan 1982), implying that most bulges went through an active galactic nucleus (AGN) phase. Galaxies and AGNs exhibit coordinated cosmic "downsizing": the star formation of massive galaxies peaks at $z \sim 2$ (Cimatti et al. 2006), and black hole (BH) growth, as traced by quasar luminosity function (Hasinger et al. 2005; Silverman et al. 2008), peaks in the same redshift range $(z=2-3)$, while the star formation of lower mass galaxies peaks at $z=1-1.5$, as do lower mass and lower luminosity AGNs (La Franca et al. 2005; Bongiorno et al. 2007). SMBH 
growth seems to be connected to galaxy growth, but the details of this mutual relation remain poorly understood (i.e., Merloni \& Heinz 2008).

To fully understand how co-evolution works requires the measurement of at least seven parameters in large samples of AGNs: (1) SMBH mass $\left(M_{\mathrm{BH}}\right.$, using broad emission line widths and optical luminosity), (2) SMBH growth rate (from bolometric luminosity), (3) galaxy mass ( $M_{\text {star }}$, via optical to infrared spectral energy distributions, SEDs, fitting), (4) star formation rate (using far-infrared or UV emission, narrow emission lines in optical spectra), (5) AGN host galaxy morphology (high-resolution imaging), (6) galaxy environment (using spectroscopic or reliable photometric redshifts), and (7) dark matter halo mass (lensing studies). To measure all the above quantities, a complete sample with spectroscopic and radio to X-ray photometric data is necessary. To assemble this kind of sample is not an easy task.

The selection of AGNs at X-ray wavelengths does not suffer from the heavy contamination by nonactive (mainly starforming) galaxies that affects infrared or optical surveys (Donley et al. 2008, 2012). Moreover, X-ray surveys are efficient in selecting low-luminosity and obscured AGNs up to equivalent hydrogen column densities, $N_{\mathrm{H}}$, of $10^{24} \mathrm{~cm}^{-2}$ (Compton thick regime). Therefore, X-ray-selected samples of AGNs, coupled with multiwavelength data, are ideal to cleanly derive the bolometric output and the accretion rate of the BHs in the majority of AGNs. For these reasons, over the last 10 years, deep and wide X-ray surveys have been carried out with the Chandra and XMM-Newton satellites to generate samples of X-ray-emitting AGNs, coupled with deep multiwavelength spectroscopic and photometric coverage to provide all the above parameters for large samples over large volumes.

Chandra and XMM-Newton contiguous extragalactic surveys are, like a wedding cake, layered in increasing depth but decreasing area (see Figure 5 of Elvis et al. 2009), from the wide/shallow XBootes survey ( $9 \mathrm{deg}^{2}$; Murray et al. 2005) to the ultra-deep/narrow survey of the 4 Ms Chandra Deep Field South (CDFS; $0.1 \mathrm{deg}^{2}$; Xue et a. 2011). The major advantage of ultra-deep surveys is that they are able to detect the X-ray emission of nonactive galaxies at medium-high redshifts (Luo et al. 2011; Lehmer et al. 2012), although they do not probe a large cosmological volume and thus contain relatively small numbers of sources at any given redshift. Larger areas are required to uniformly sample the parameter space (luminosity and redshift) (Brusa et al. 2009; Civano et al. 2011), obtain large samples of rare sources (e.g., recoiling $\mathrm{SMBH}$; Civano et al. 2010) and measure large-scale structures in the universe (Hickox et al. 2011; Allevato et al. 2011; Capak et al. 2011) and hence determine the relationship between galaxy evolution and local environmental density. For a complete picture, it is clearly necessary to explore the parameter space between the ultra-deep and ultra-wide surveys. Eventually, the best approach to trace the full population of AGNs at all redshifts and all luminosities is to merge the data coming from all the layers of the wedding cake (see, e.g., Fiore et al. 2012).

The Chandra COSMOS project (C-COSMOS; Elvis et al. 2009, Paper I hereafter) hits a sweet spot among the wedding cake layers (Figure 5 in Elvis et al. 2009): deep enough $(1.9 \times$ $10^{-16} \mathrm{erg} \mathrm{cm}^{-2} \mathrm{~s}^{-1}$ in the $0.5-2 \mathrm{keV}$ band) to find obscured AGNs with optical galaxy continua and wide enough $\left(0.9 \mathrm{deg}^{2}\right)$ to have large samples (1761 X-ray point-like sources) and find unusual, rare objects (Fiore et al. 2009; Civano et al. 2010, 2012; Capak et al. 2011). Yet, the C-COSMOS sources are bright enough that virtually all X-ray sources can be identified and followed up across their SEDs, especially with optical or nearIR spectroscopy, using the vast Cosmic Evolutionary Survey (COSMOS; Scoville et al. 2007) multiwavelength photometric and spectroscopic database (Schinnerer et al. 2007; Sanders et al. 2007; Taniguchi et al. 2007; Capak et al. 2007; Koekemoer et al. 2007; Zamojski et al. 2007; Lilly et al. 2007; Trump et al. 2007). Largely thanks to the low background of Chandra, the CCOSMOS survey reaches four times fainter fluxes than XMMCOSMOS (Hasinger et al. 2007; Cappelluti et al. 2009; Brusa et al. 2010) in both the $0.5-2 \mathrm{keV}$ and $2-8 \mathrm{keV}$ bands. The heavily overlapping "dense tiling" observation strategy, now also used in other Chandra surveys (e.g., Drake et al. 2009), combined with the subarcsecond on-axis Chandra point-spread function (PSF; Weisskopf et al. 2002), provides a rather uniform sensitivity in C-COSMOS (see Paper I and Puccetti et al. 2009, hereafter Paper II, for details). Thus, the high-resolution area is maximized to resolve sources with a subarcsecond position accuracy, a key ingredient for the best identification of the optical counterparts of the X-ray sources, to find AGN pairs (Silverman et al. 2011; E. Cunningham \& M. Kriek in preparation) and offnuclear sources (Mainieri et al. 2010).

Historically, various classes of X-ray emitters have been characterized by different values of the X-ray to optical flux ratio (hereafter $\mathrm{X} / \mathrm{O}=\log \left(f_{X} / f_{\text {opt }}\right)$ ) which provides a first indication of the source classification (Maccacaro et al. 1988). Originally, the $\mathrm{X} / \mathrm{O}$ ratio was defined in the soft band of the Einstein Observatory $(0.3-3.5 \mathrm{keV})$ at fluxes of $10^{-13}$ to $10^{-11} \mathrm{erg} \mathrm{cm}^{-2} \mathrm{~s}^{-1}$ and later modified to the lower energy ROSAT band (0.1-2.4 keV); the majority of spectroscopically identified AGNs in soft X-ray surveys were characterized by $\mathrm{X} / \mathrm{O}=0 \pm 1$.

With the advent of Chandra and XMM-Newton, the same relation has been used in harder bands (Hornschemeier et al. 2001; Alexander et al. 2001; Fiore et al. 2003; Della Ceca et al. 2004; Cocchia et al. 2007). The optical identification of the sources found in deep and medium surveys (Chandra Deep Fields, Hellas2XMM, XMM-COSMOS) confirms the trend observed at bright fluxes but also shows a large scatter around the median value in both the hard and soft bands (Brandt \& Hasinger 2005). Obscured AGNs $\left(N_{\mathrm{H}}>10^{22} \mathrm{~cm}^{-2}\right)$ tend to populate the upper part of this plot and have $\mathrm{X} / \mathrm{O}>1$ (Perola et al. 2004; Civano et al. 2005; Brusa et al. 2010), while normal galaxies, detected at very faint $\mathrm{X}$-ray fluxes, have $\mathrm{X} / \mathrm{O}<-2$ (Xue et al. 2011). In addition to obscured AGNs, unobscured $\mathrm{X}$-ray-Bright Optically Normal Galaxies also have $\mathrm{X} / \mathrm{O}>1$ (e.g., Comastri et al. 2002; Civano et al. 2007; Hornschemeier et al. 2003; Trump et al. 2009b). The lack of information for faint $\mathrm{X}$-ray and optical sources, plus their high $\mathrm{X} / \mathrm{O}$ ratio, led the scientific community to name these kinds of sources "extreme" or "unconventional" (Comastri et al. 2003; Mignoli et al. 2004), in particular when the $\mathrm{X} / \mathrm{O}$ is defined in the hard $\mathrm{X}$-ray band.

In this paper (Paper III), we present the catalog of optical and infrared counterparts of Chandra COSMOS sources, presented in Paper I, and we analyze some of their observed properties. The paper is organized as follows: Section 2 describes the data sets used in this work, Sections 3 and 4 explain the details of the method used for the identifications and the results obtained, in Section 5 the positional and magnitude distributions are presented, in Section 6 we show the spectroscopic and photometric redshifts along with the spectral and SED classification, in Section 7 we present the identification catalog, in Section 8 the optical and infrared properties are compared 
with the X-ray ones, in Section 9 we summarize the results of this work.

We assume a cosmology with $H_{0}=70 \mathrm{~km} \mathrm{~s}^{-1} \mathrm{Mpc}^{-1}$, $\Omega_{M}=0.3$, and $\Omega_{\Lambda}=0.7$. The AB magnitude system is used in this paper if not otherwise stated.

\section{IDENTIFICATION DATA SETS}

The C-COSMOS X-ray source catalog used in this work comprises $1761 \mathrm{X}$-ray point sources detected down to a maximum likelihood threshold $\operatorname{detml}=10.8$ in at least one band (0.5-2, $2-8$, or $0.5-8 \mathrm{keV})$. This likelihood threshold corresponds to a probability of $\sim 5 \times 10^{-5}$ that a catalog source is instead a background fluctuation (Paper II). At this threshold, the flux limit reached in the survey is $5.7 \times 10^{-16} \mathrm{erg} \mathrm{cm}^{-2} \mathrm{~s}^{-1}$ in the full band $(0.5-10 \mathrm{keV}), 1.9 \times 10^{-16} \mathrm{erg} \mathrm{cm}^{-2} \mathrm{~s}^{-1}$ in the soft band $(0.5-2 \mathrm{keV})$, and $7.3 \times 10^{-16} \mathrm{erg} \mathrm{cm}^{-2} \mathrm{~s}^{-1}$ in the hard band (2-10 keV, extrapolated from the observed 2-7 keV band). Of the 1761 sources, 922 are detected in all three bands (full, soft, hard), 474 are detected in the full and the soft band, 257 are detected in the full and the hard band, 73 only in the full, 32 only in the soft, and 3 only in the hard band. Upper limits ( $3 \sigma$ values) in each band have been computed for the sources detected in only one or two bands (see Paper II).

We identify counterparts of the C-COSMOS sources in the $i$ band $(0.76 \mu \mathrm{m})$ using the Subaru photometric catalog (Capak et al. 2007, hereafter "optical catalog"), in the $K$ band $(2.15 \mu \mathrm{m})$ using the Canada-France-Hawaii Telescope (CFHT)/Megacam catalog (McCracken et al. 2010) and at $3.6 \mu \mathrm{m}$ using the Spitzer IRAC catalog (Sanders et al. 2007). The full optical catalog contains about 1.5 million sources down to a magnitude limit $i \sim 27(\mathrm{AB})$ at a signal-to-noise ratio of $((\mathrm{S} / \mathrm{N})>5)$. The $K$-band catalog contains $\sim 500,000$ sources detected at $\mathrm{S} / \mathrm{N}>5$ to a magnitude limit of 23.5 (AB). The IRAC catalog contains $\sim 350,000$ sources at $3.6 \mu \mathrm{m}$ to 23.9 (AB, corresponding to $\sim 1 \mu \mathrm{Jy}$ ) at an $\mathrm{S} / \mathrm{N}>5$.

In the second part of the identification process, we also made use of the Advanced Camera for Surveys (ACS)/ Hubble Space Telescope (HST) images of the COSMOS field (Scoville et al. 2007; Koekemoer et al. 2007) to visually check our identifications, taking advantage of the ACS PSF, of the accuracy of the positions, and of the depth of the observations $\left(I_{\mathrm{F} 814 \mathrm{~W}} \sim 27.8 \mathrm{AB} \mathrm{mag}, 5 \sigma\right.$ for an optimally extracted point source). A new, revised photometric catalog, which includes the photometry in all the 25 optical/NIR broad-, intermediate-, and narrow-band filters, with improved source detection ${ }^{20}$ has been used to match the final identifications.

\section{X-RAY SOURCE IDENTIFICATION METHOD}

\subsection{Method}

We used the likelihood ratio technique of Sutherland \& Saunders (1992), following the procedure described by Brusa et al. (2005), which when applied to the identification of the XMM-COSMOS sample (Brusa et al. 2007, 2010) yielded a percentage of "reliable identifications" greater than $80 \%$. With respect to simpler matching approaches, based only on the positional offset between sources in different catalogs, the

\footnotetext{
20 The catalog is publicly available at http://irsa.ipac.caltech.edu/data/ COSMOS/tables/photometry/ and supersedes the catalog reported by Capak et al. (2007). The photometry is computed at the position of the $i^{*}$-band image, using SExtractor (Bertin \& Arnouts 1996) in dual mode. The same catalog is used in Ilbert et al. $(2009,2010)$ and Salvato et al. $(2009,2011)$.
}

likelihood ratio technique has the advantage of taking into account, for each possible counterpart, the probability that it is a real or a spurious identification, allowing a much better statistical characterization of the final sample of identifications. This powerful statistical method has become common recently and has been applied first to radio surveys (Ciliegi et al. 2005) and to several medium and faint Chandra and XMM-Newton surveys (Cardamone et al. 2008; Laird et al. 2009; Aird et al. 2010; Luo et al. 2010; Brusa et al. 2010; Xue et al. 2011; Rovilos et al. 2011).

Identifying the true counterpart of Chandra sources is easier than for XMM-Newton because of the narrower Chandra PSF, therefore excellent positional accuracy (on average $<0$ '. 5 ; Paper II), and the tighter Chandra encircled energy fraction ${ }^{21}$ (typically $90 \%$ at $0^{\prime \prime} .5$ on-axis to $5^{\prime \prime}$ at $12^{\prime}$ off-axis; Weisskopf et al. 2002). X-ray sources at C-COSMOS fluxes have, on average, redder colors than brighter X-ray sources (Brandt \& Hasinger 2005) and thus the likelihood ratio in near-infrared bands should produce a high rate of identifications. In this paper, we therefore give equal weight to the redder bands ( $K$, $3.6 \mu \mathrm{m})$ as to the optical $(i)$, so that we can maximize the number of identified sources, and also compare the identification efficiency at different wavelengths. This approach is possible only because the depth of the $K$ and IRAC $3.6 \mu \mathrm{m}$ COSMOS observations nicely matches the X-ray depth for a typical AGN SED (e.g., Elvis et al. 1994; Elvis et al. 2012). Deep X-ray surveys (e.g., 2 or more megaseconds in the CDFS) reveal, besides a population of bright normal galaxies, a population of fainter X-ray sources which will be hard to follow up across the SED with the currently available optical or near-IR capabilities.

We can summarize the four steps of our identification procedure as follows.

1. Run the likelihood ratio technique separately in the three different bands ( $i, K$, and $3.6 \mu \mathrm{m}$; Section 3.2).

2. Find appropriate parameters (threshold, normalization) in order to identify the sources and define the level of confidence of the association (Section 3.3).

3. Combine the results obtained in the three bands (Section 4).

4. Confirm the uncertain cases, visually checking the HST ACS image at the position of the X-ray source (Sections 4.1 and 4.3).

\subsection{Likelihood Ratio Definition}

For a given optical or infrared candidate with magnitude $m$ and positional offset $r$ from the X-ray source position, the likelihood ratio LR is defined as the ratio between the probability that the source is the correct identification and the corresponding probability for a background, unrelated object:

$$
\mathrm{LR}=\frac{q(m) f(r)}{n(m)},
$$

where $q(m)$ is the expected magnitude $m$ distribution function (normalized to one) of the real optical counterpart candidates, $f(r)$ is the probability distribution function of the positional errors (assumed to be a two-dimensional Gaussian), and $n(m)$ is the surface density of background objects with magnitude $m$.

The distribution of the local background objects, $n(m)$, was computed from each of the three input catalogs using the objects within a $5^{\prime \prime}-30^{\prime \prime}$ annulus around each X-ray source. We chose a $5^{\prime \prime}$ inner radius in order to avoid the presence of true counterparts

\footnotetext{
21 The encircled energy fraction is the two-dimensional integral of the PSF.
} 
in the background distribution, and a $30^{\prime \prime}$ outer radius to exclude the counterparts of other nearby X-ray sources. In the case of $\mathrm{X}$-ray source pairs (178 pairs with relative distances $<15^{\prime \prime}$ and 400 with distances $<30^{\prime \prime}$ ), the outer radius could contain the counterpart of a nearby X-ray source. However, the number of background sources in the annuli is sufficiently large $(\sim 100$ sources on average in the $K$ band down to $22 \mathrm{mag}$ and $\sim 300$ sources in the $i$ band down to $25 \mathrm{mag}$ ) to make the presence of a nearby object's counterpart unimportant.

The function $q(m)$ has been estimated from our data as follows. In this work, we neglect the possible X-ray flux dependence of $q(\mathrm{~m})$ and assumed a universal optical/infrared magnitude distribution for all X-ray sources. We first computed $q^{\prime}(m)=$ [number of sources with magnitude $m$ within $\left.1^{\prime \prime}\right]$ [expected number of background sources with magnitude $m$ in a $1^{\prime \prime}$ circle]. The choice of a $1^{\prime \prime}$ radius is dictated by the requirement of maximizing the statistical significance of the overdensity around the X-ray sources. A smaller radius would include in the analysis only a fraction of the true identifications and the $q(m)$ distribution would be more affected by Poissonian noise. A larger radius would increase the number of background sources.

In Figure 1, the observed magnitude distribution of the objects in the three catalogs $(i, K$, and $3.6 \mu \mathrm{m})$ within a radius of $1^{\prime \prime}$ around each X-ray source (solid histogram) is plotted together with the expected distributions of background objects in the same area (dashed histogram). The difference between these two distributions is the expected distribution of the counterparts $\left(q^{\prime}(m)\right.$, red curve) before normalization. The distribution of background sources in the $i$ band (Figure 1, top panel) is more prominent than in the $K$ or $3.6 \mu \mathrm{m}$, because of the fainter magnitude limit of the optical catalog (the number counts of galaxies in the $i$ band strongly increase at faint magnitude, see, e.g., Ilbert et al. 2010).

Then, $q(m)=$ const $\times q^{\prime}(m)$ is normalized such that $\int_{-\infty}^{+\infty} q(m) d m=1$. In practice, since we have a magnitude limit $m_{\text {lim }}$, we normalized in such a way that $Q=\int_{m} q(m) d m=$ (number of X-ray sources identified in each band/the total number of sources of the X-ray sample). More details are given in Section 3.3.

For the probability distribution of positional errors, $f(r)$, we adopted a Gaussian distribution with standard deviation, $\sigma=\sqrt{\sigma_{X}^{2}+\sigma_{\mathrm{opt}}^{2}}$, where $\sigma_{\mathrm{opt}}$ is the positional uncertainty in the three optical and infrared bands. $\sigma_{X}$ is taken from Column 5 in Table 7 of Paper I (see also Figure 12 in Paper II). The adopted $\sigma_{\text {opt }}$ is 0.2 for the $K$ band (McCracken et al. 2010), $0^{\prime} .3$ in the $i$ band (Capak et al. 2007), and 0.'5 at $3.6 \mu \mathrm{m}$ (Sanders et al. 2007).

Having determined the values of $q(m), f(r)$, and $n(m)$ for each band, using Equation (1) we computed the LR value for all the sources within $5^{\prime \prime}$ of the $1761 \mathrm{X}$-ray centroids in each of the three catalogs (a total of 10,972 sources for the $i$ band, 2992 sources for the $K$ band, and 2604 for the IRAC $3.6 \mu \mathrm{m}$ band).

\subsection{Threshold Choices}

Once the LR values have been computed for all the candidates, one has to choose the best likelihood threshold value $\left(L_{\mathrm{th}}\right)$ for LR to discriminate between spurious and real identifications. The choice of $L_{\text {th }}$ depends on two factors: first, $L_{\text {th }}$ should be small enough to avoid missing many real identifications, so that the completeness of the sample is high; second, $L_{\text {th }}$ should be
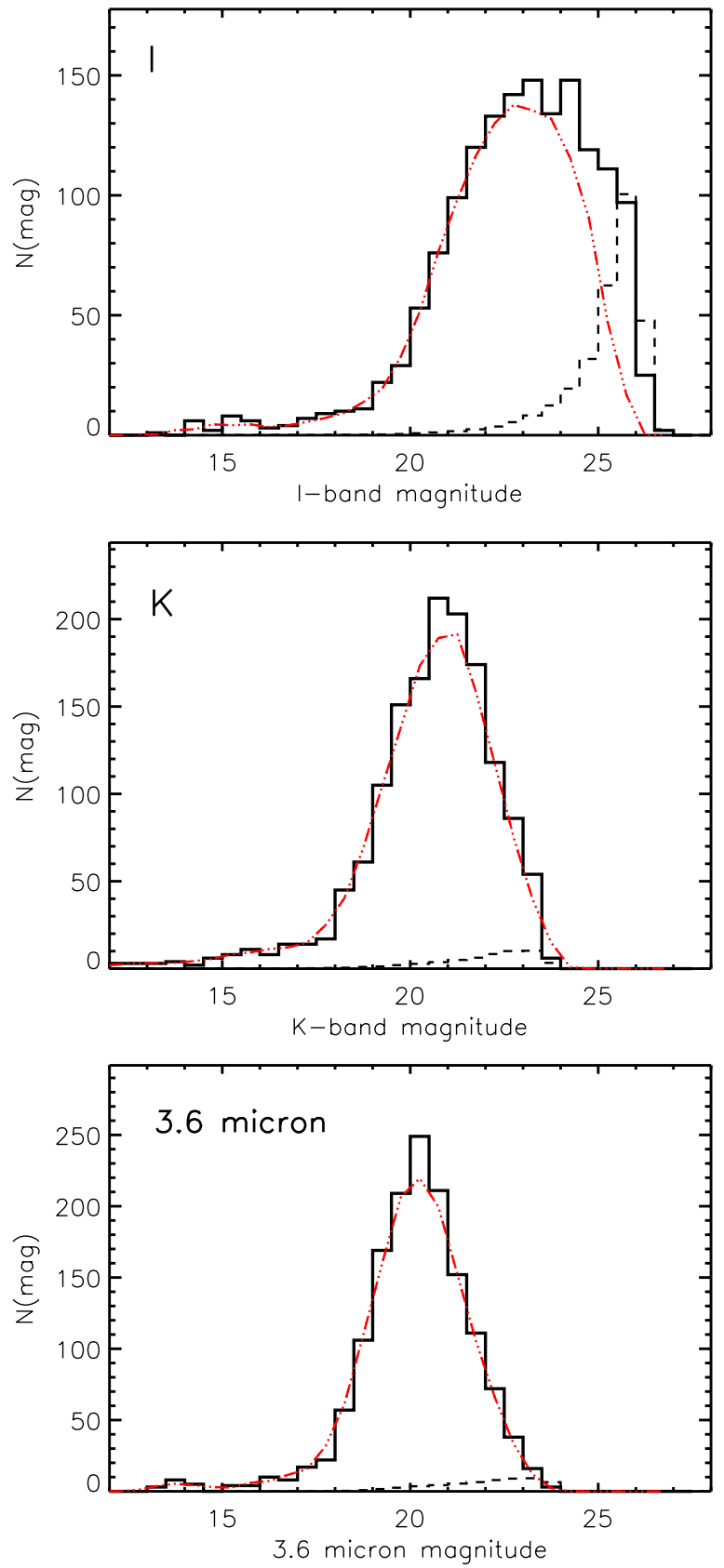

Figure 1. Observed $\mathrm{AB}$ magnitude distribution of all the $i$-band, $K$-band, and $3.6 \mu \mathrm{m}$ band (from top to bottom) objects within a radius of $1^{\prime \prime}$ around the 1761 $\mathrm{X}$-ray sources (solid black histogram) together with the expected distribution of background objects $(n(m)$, black dashed histogram). The difference between the two distributions (red dash-dotted line) corresponds to the expected magnitude distribution of counterparts $\left(q^{\prime}(m)\right.$, smoothed here for plotting purposes).

(A color version of this figure is available in the online journal.)

large enough to keep the number of spurious identifications low, in order to increase the reliability of the identification.

The reliability takes into account the possibility of having multiple optical candidates for the same X-ray source. The reliability $R_{j}$ for object $j$ being the correct identification is

$$
R_{j}=\frac{(\mathrm{LR})_{j}}{\Sigma_{i}(\mathrm{LR})_{i}+(1-Q)},
$$

where the sum is over the set of all candidates for source $j$. The reliability parameter $(R)$ for the total sample is defined as the ratio between the sum of the reliability of all the sources 
Table 1

Identification Percentages in Each Band before (Columns 3, 5, 7) and after (Columns 4, 6, 8) the Match between the Three Catalogs, the Visual Checks, and after Recovering the Faint Sources

\begin{tabular}{|c|c|c|c|c|c|c|c|c|c|}
\hline & Class & $\begin{array}{c}i \\
\text { Before }\end{array}$ & $\begin{array}{c}i \\
\text { After }\end{array}$ & $\begin{array}{c}K \\
\text { Before }\end{array}$ & $\begin{array}{c}K \\
\text { After }\end{array}$ & $\begin{array}{l}3.6 \mu \mathrm{m} \\
\text { Before }\end{array}$ & $\begin{array}{c}3.6 \mu \mathrm{m} \\
\text { After }\end{array}$ & Total \% & Total Number \\
\hline (1) & Secure ID & $84.8 \%$ & $84.1 \%$ & $90.1 \%$ & $89.7 \%$ & $95.6 \%$ & $94.8 \%$ & $96.3 \%$ & 1708 \\
\hline (2) & Ambiguous ID & $3.7 \%$ & $3.7 \%$ & $2.7 \%$ & $2.6 \%$ & $1.3 \%$ & $1 \%$ & $2 \%$ & 24 \\
\hline (3) & Subthreshold ID & $9.2 \%$ & $6.1 \%$ & $4.2 \%$ & $2.3 \%$ & $1.8 \%$ & $0.8 \%$ & $1.1 \%$ & 19 \\
\hline (4) & Unidentified & $1 \%$ & $1.8 \%$ & $3.1 \%$ & $3.6 \%$ & $1.3 \%$ & $3.4 \%$ & $0.6 \%$ & 10 \\
\hline (5) & Retrieved & & $3.3 \%$ & & $1.8 \%$ & & & & \\
\hline
\end{tabular}

Note. The final percentages are reported in Column 9.

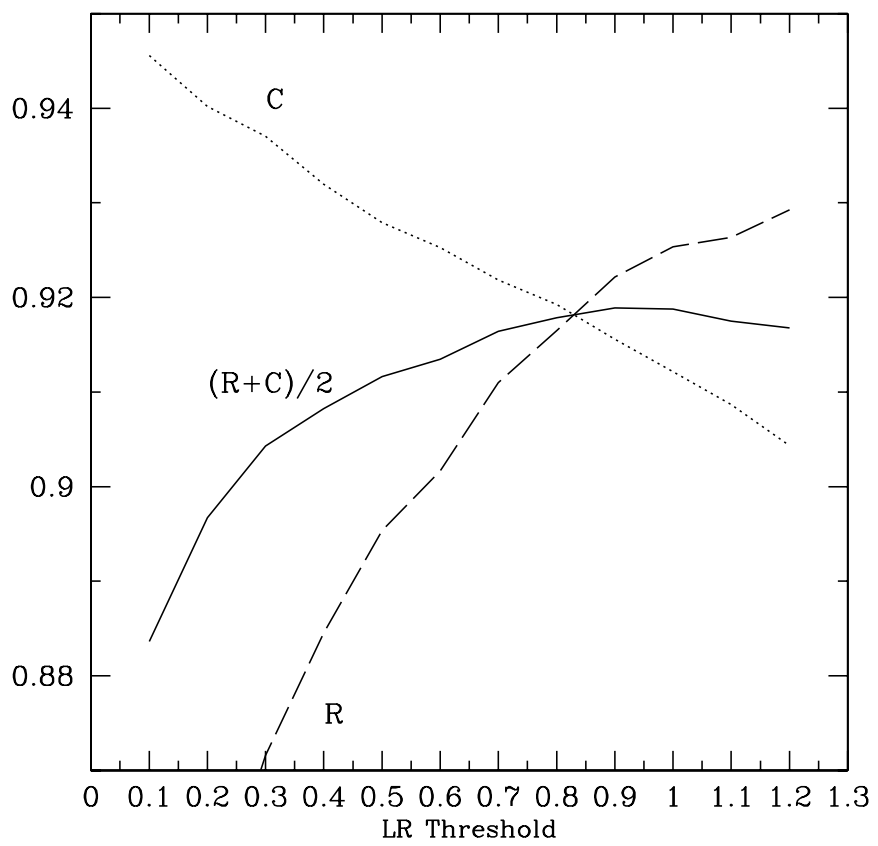

Figure 2. Completeness (dotted line) and reliability (dashed line) parameters and $(C+R) / 2$ (solid line) of our sample in the $K$ band with a normalization value $N=0.9$. The $L_{\text {th }}$ chosen corresponds to the value where the $(C+R) / 2$ (solid line) quantity is maximized but still close to the intersection of the three curves.

identified as possible counterparts and the total number of sources with LR $>L_{\text {th }}\left(R=N_{\mathrm{ID}} / N_{\mathrm{LR}>L_{\mathrm{th}}}\right)$.

The completeness parameter $(C)$ of the total sample is defined as the ratio between the sum of the reliability of all the sources identified as possible counterparts and the total number of X-ray sources $\left(C=N_{\mathrm{ID}} / N_{X}\right)$.

Following Brusa et al. (2007), we defined $L_{\text {th }}$ as the likelihood ratio which maximizes the quantity $(R+C) / 2$. We applied this criterion to the three input catalogs $(i, K$, and $3.6 \mu \mathrm{m})$ and the resulting $L_{\mathrm{th}}$ are $0.4,0.9$, and 0.47 , respectively.

The corresponding sample completeness and reliability are $C=0.85$ and $R=0.88$ for $i, C=0.90$ and $R=0.92$ for $K$, and $C=0.96$ and $R=0.96$ for $3.6 \mu \mathrm{m}$. As an example, in Figure 2, $C, R$, and $(R+C) / 2$ versus $L_{\text {th }}$ are reported for the $K$-band catalog, using 0.9 as normalization for $q^{\prime}(m)$ to convert into $q(m) .(R+C) / 2$ is quite flat over $0.7<L_{\text {th }}<1.2$. The value chosen corresponds to the point where $(C+R) / 2$ is maximized and it is close to where $C, R$, and $(C+R) / 2$ curves intersect.

It is possible to make a direct comparison with the likelihood threshold values used by Brusa et al. (2007) for XMM-COSMOS because the same input optical catalog was used. Thanks to the better Chandra positional accuracy, at the same LR threshold in the $i$ band $\left(L_{\mathrm{th}}=0.4\right)$ a higher completeness and reliability can be achieved in Chandra than in $X M M(C=0.77$ and $R=0.69$ in Brusa et al. 2007, versus $C=0.85$ and $R=0.88$ here). A lower LR threshold in the $i$ band was used for the final XMM-COSMOS catalog $\left(L_{\text {th }}=0.26\right.$; Brusa et al. 2010); however, its completeness and reliability cannot be compared with those of C-COSMOS, as the Chandra COSMOS associations have been used to resolve some ambiguous identifications in the XMM-COSMOS catalog.

\section{X-RAY SOURCE IDENTIFICATION RESULTS}

In this section we present the various steps that we followed to define the best associations after running the likelihood ratio analysis. We first used the output of the likelihood ratio, then we merged the results in the three bands, inspected the associations, and listed the final counterparts.

Following the same approach used for XMM-COSMOS identifications (Brusa et al. 2007, 2010), the X-ray sources have been divided into four different classes (see Table 1).

1. Secure. Sources for which the likelihood procedure is able to find only one counterpart with LR $>L_{\text {th }}$. The vast majority of the sources belong to this class; examples are given in panels 1-3 in Figure 3 .

2. Ambiguous. Sources with more than one counterpart above the threshold (panels 4-6 in Figure 3).

3. Subthreshold. Sources with no counterpart with LR $>L_{\text {th }}$ but with a possible counterpart with $\mathrm{LR}<L_{\text {th }}$ within $5^{\prime \prime}$ (panels 10-12 in Figure 3).

4. Unidentified. Sources with no counterpart in each given catalog, even below the threshold, within $5^{\prime \prime}$ from the X-ray centroid (panels 7-9 in Figure 3).

We anticipate here another small class (5) retrieved, which is not a direct output of the likelihood procedure, and which has been introduced after the visual inspection and is discussed later (see Section 4.3).

\subsection{Ambiguous Source Resolution}

In the class of ambiguous sources, there are cases with more than one possible counterpart above the LR threshold. For example, in the $K$ band, there are $36 \mathrm{X}$-ray sources with two possible counterparts, 4 sources with three possible counterparts, none with four possible counterparts, and 1 source with five possible counterparts, all of the counterparts being above $L_{\mathrm{th}}$. Even though the X-ray sources in Elvis et al. (2009) are all unresolved point sources that show no evidence for extended emission, some of the sources with multiple counterparts could be associated with X-ray groups. However, this possibility has not been explored and we assumed that all 


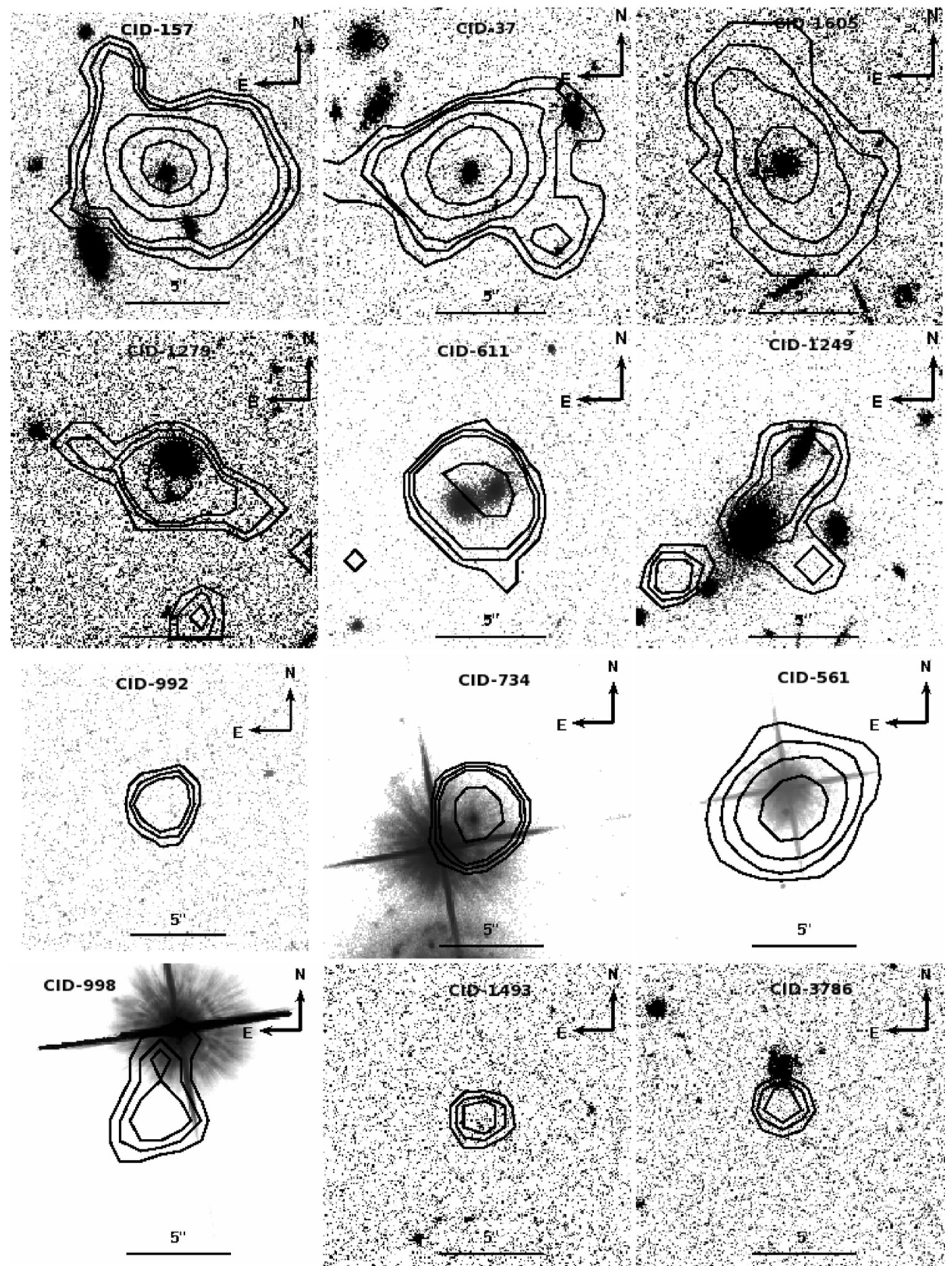

Figure 3. Examples of identifications. X-ray contours are overlaid on the ACS images. Secure identified sources (1-3): CID-157, CID-37, CID-1605; Ambiguous sources (4-6): CID-1279, CID-611, CID-1249. The two possible counterparts are visible in the inner X-ray contour; Unidentified sources (7-9): CID-992 is a truly empty field at all wavelengths, and CID-734 and CID-561 are point-like bright sources very close to a bright star so it is not possible to derive their photometry. Subthreshold sources (10-12): CID-998 is a bright star at a large distance from the X-ray centroid, CID-1493 is a very faint source $\left(i_{\mathrm{AB}}=26.43\right)$, and CID-3786 is a bright optical source but not very close to the $\mathrm{X}$-ray centroid.

the X-ray sources are associated with a single optical/infrared counterpart. We also inspected the location of ambiguous sources on the field, but their size is consistent with a single point-like source.
In order to resolve some of the ambiguous cases, we computed the distributions of the ratios between the LR of the highest LR counterpart and the other counterparts for the same X-ray source. In Figure 4, the histogram of the ratio of LRs of the first 

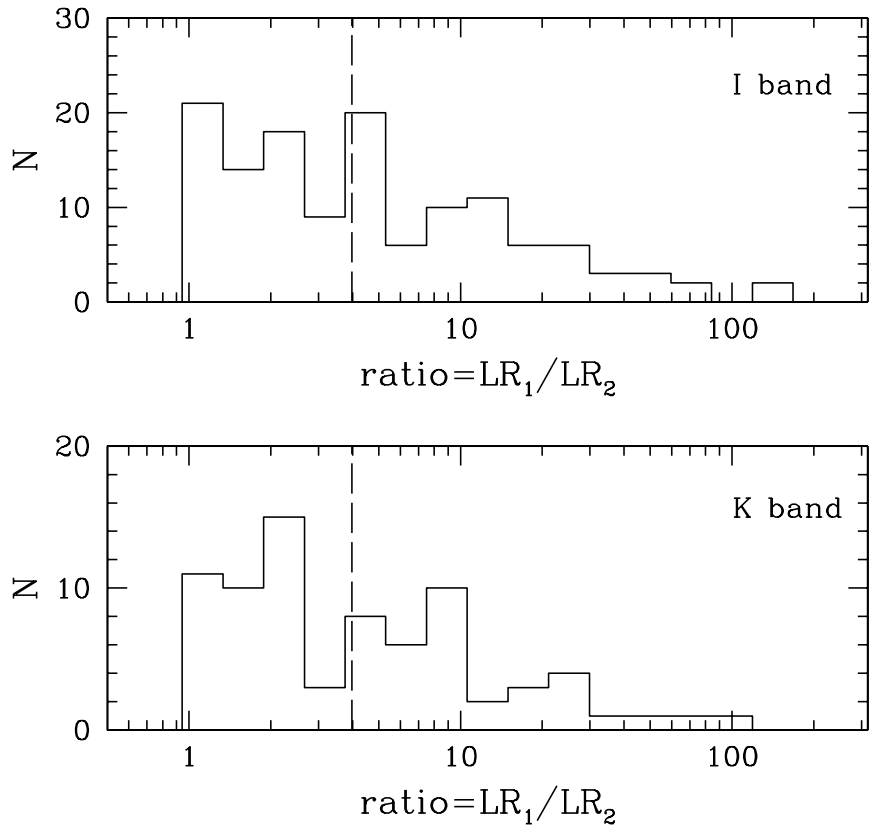

Figure 4. Histograms of the ratio of LRs of the first and the second possible counterparts for the ambiguous sources in the $i$ (top panel) and in the $K$ (bottom panel) band. The dashed lines represent the median value of the LR1/LR2 distribution. We adopt this value to solve some of the ambiguities.

and the second possible counterparts is reported for the $i$ - and $K$-band ambiguous sources. A high ratio strongly suggests that the highest likelihood counterpart is the correct one. In both $i$ and $K$ bands, if the ratio is above the median value (LR1/LR2 $=4$ in both bands, dashed lines in Figure 4), we define the highest LR object as the secure identification. This method has been applied before in M. Brusa et al. (2007, private communication). Using this criterion, by definition, $50 \%$ of the ambiguous cases in the $K$ and $i$ bands, respectively, are solved. The sources with two possible counterparts are flagged accordingly in the catalog in the identification flag column (see Section 7). The final numbers of ambiguous sources in each band after this resolution are reported in Table 1.

\subsection{Identification Rates}

In Table 1 (Columns 3, 5, and 7), we report the fraction of sources in each of the classes described above for each band obtained from the output of the LR analysis.

The $K$-band catalog identification returns a higher identification rate of secure sources than the $i$-band identification $(90 \%$ versus $85 \%)$, fewer sources below $L_{\text {th }}(4.2 \%$ versus $9.2 \%)$ and fewer ambiguous sources $(2.7 \%$ versus $5 \%)$. The differences are due to the different depths of the $i$ - and $K$-band catalogs. The $K$-band catalog is deep enough to recover most of the counterparts, showing the good coupling of X-ray and $K$ fluxes, without being too deep to introduce ambiguities and increase the number of spurious associations. For comparison, at a similar depth both in the optical and in the X-ray, $82 \%$ of sources have been successfully identified in the AEGIS-X survey (Laird et al. 2009), while in the CDFS, at fainter fluxes, the fractions of secure identifications in both the optical and $K$ band are slightly lower (74\% and $85 \%$; Luo et al. 2010).

The $3.6 \mu \mathrm{m}$ identification rate is the highest of the three (95\%). The depths of the COSMOS $3.6 \mu \mathrm{m}$ and $K$-band catalogs are nearly the same, but $3.6 \mu \mathrm{m}$ favors the detection of red objects even more than the $K$ band and thus retrieves those unde- tected in the optical, leading to the increased identification rate. Similar $3.6 \mu \mathrm{m}$ identification rates (98\% and 94\%) have been found for the ECDFS and the AEGIS-X samples (Cardamone et al. 2008; Laird et al. 2009) at the same X-ray flux limit, but at a magnitude limit of $24.4 \mathrm{AB}(5 \sigma)$ and $23.8 \mathrm{AB}$, respectively. A somewhat lower identification rate $(89 \%)$ has been found in the CDFS (2 Ms catalog; Luo et al. 2010) at similar IRAC depth.

However, the blending and confusion of IRAC sources, due to the larger PSF (1".7 at $3.6 \mu \mathrm{m})$, might contribute to spuriously increasing the identification rate. To quantify this effect, we ran the likelihood procedure assuming the same background source distribution used above, the same $L_{\text {th }}$ (this is a conservative choice given that a fainter sample implies a lower $L_{\text {th }}$ to maximize completeness and reliability), and decreasing the flux of the objects with $5^{\prime \prime}$ from the X-ray position by a factor of two. The number of subthreshold sources increases to $5.5 \%$, which is still a low number and comparable to the results obtained in the $K$ band. We thus believe that, even if all the $3.6 \mu \mathrm{m}$ sources in the sample are contaminated by bright neighbors, and their flux is somehow fainter, the secure source fraction would still be of the order of $\sim 90 \%$. According to this result, the blending problem, which might still be important for SED fitting, does not invalidate the results of the likelihood ratio analysis.

Among the sources with LR $>L_{\text {th }}$ (secure and ambiguous), we estimated the number of spurious associations in each band. This number corresponds to the difference between the number of X-ray sources with at least one identification with $L R>L_{\text {th }}$ and the expected number of real identifications obtained adding the reliability of all the objects with $\mathrm{LR}>L_{\mathrm{th}}$. The obtained percentages are $6 \%$ in the $i$ band, $1 \%$ in the $K$ band, and $2 \%$ at $3.6 \mu \mathrm{m}$.

\subsection{Cross-wavelength Matching of Identifications}

The identifications from the three catalogs have been compared in order to check the consistency among the counterparts. We performed a positional cross-correlation first between the $K$ and $i$ bands, and then the $K$ and $3.6 \mu \mathrm{m}$ results. While checking the results of the cross-correlation, we visually inspected the associations by using HST/ACS (filter F814W), $K$, IRAC $(3.6 \mu \mathrm{m})$, and Chandra $15^{\prime \prime} \times 15^{\prime \prime}$ cutouts around each X-ray source. The ACS cutouts have been used because of the better subarcsecond PSF with respect to the ground-based $i$-band images. We also made use of X-ray contour levels, produced with the CIAO tool DMCONTOUR ${ }^{22}$ on the Chandra mosaic (as shown in Figure 3).

First, the $K$ - and $i$-band results have been cross-matched using a radius of 0.7 , which maximizes the number of associations without introducing unreliable matches: $88 \%$ of the X-ray sources are associated with the same counterpart (86.4\% above the threshold in each band), while $\sim 7 \%$ of the sources have different associations in the two bands.

In most of the latter cases, the $i$-band associations are subthreshold while the $K$-band ones are not, suggesting a misidentification in the $i$ band. By visually inspecting the cutouts, we verified that in almost all these cases the sources associated with the $K$-band counterparts are not present in the input optical catalog because of their faintness or low $\mathrm{S} / \mathrm{N}$. Therefore, the association with the $K$-band counterparts has been taken as correct and the initial $i$-band association discarded. We retrieved the $i$ magnitudes of the $K$-band counterpart from the original optical catalog (with no cut in $\mathrm{S} / \mathrm{N}$ ) when available.

\footnotetext{
22 http://cxc.harvard.edu/ciao/ahelp/dmcontour.html
} 
This process leads to a decrease of the number of the subthreshold sources in the $i$ band and the definition of a new class (5) "retrieved." When the different associations in the two bands were both secure, we used the $3.6 \mu \mathrm{m}$ associations, the cutouts, and the X-ray contour levels to help the identification, and in most of the cases $(>90 \%)$ the IRAC $3.6 \mu \mathrm{m}$ band association agreed with the $K$-band counterpart, strengthening the identification.

Of the remaining $\sim 5 \%$ of the sources, $2 \%$ are ambiguous objects in both bands with a separation between the two possible counterparts smaller than the Chandra PSF at the source position, while $3 \%$ are objects unidentified in one or both bands.

Second, the $K$ and $3.6 \mu \mathrm{m}$ results have been matched using the same 0.7 radius. A good agreement is found: $96 \%$ of the sources with a counterpart in both bands are associated with the same source. Among the sources for which we do not find a common counterpart between the two bands we find the same behaviors as above. There are objects classified as subthreshold in the $K$ band and secure at $3.6 \mu \mathrm{m}$ which are replaced by faint $K$-band sources at the $3.6 \mu \mathrm{m}$ positions. These sources have been found in the original $K$-band catalog and included as retrieved in the identification list. The original subthreshold $K$-band counterpart has been then discarded. There are also sources for which a secure association was found in both bands but these do not coincide. We visually checked the cutouts for these sources and we decided which counterpart to prefer with the help of the $i$-band associations and the X-ray contours levels.

We then performed a visual inspection of all the sources, according to the different identification classes. At this stage the $3.6 \mu \mathrm{m}$ secure association fraction decreases in favor of the unidentified sources because some of them $(<1 \%)$, due to the larger PSF, were wrongly associated with bright close-by sources (stars or galaxies) and thus are not real counterparts associated with the $i$ - and $K$-band sources.

At this stage, we also used the revised version of the optical catalog, as explained in Section 2. Among other improvements, this new version of the catalog accounts for correctly deblended faint sources close to bright/saturated ones (see more details in the changes in the header of the photometric catalog of Ilbert et al. 2009). ${ }^{23}$

\subsection{Summary of Identifications}

The fraction of sources in the three bands for each class, after correlating the LR outputs and the visual checks, is reported in Table 1 (Columns 4, 6, 8). We also report the percentage of counterparts retrieved in the $K$-band and $i$-band catalogs with a magnitude below the formal $5 \sigma$ limit of the input catalogs.

We assigned a final identification flag that summarizes the results in the three bands. The final percentages and total numbers per class are reported in the last two columns of Table 1. We use:

1. A secure identification if the source is a secure identification in at least one band (1708 sources),

2. An ambiguous identification if the source is an ambiguous identification in at least two bands (24 sources),

3. A subthreshold identification if the source is in this class in all three bands (19 sources), and

4. An unidentified source if it is not identified in any of the three bands (10 sources).

23 Available at http://irsa.ipac.caltech.edu/data/COSMOS/tables/redshift/
Table 2

Number of Objects Detected in All Combinations of 3, 2, or 1 Bands and Percentage

\begin{tabular}{lrr}
\hline \hline Band & Number of Sources & $\%$ \\
\hline$i+K+3.6 \mu \mathrm{m}$ & 1652 & 93.9 \\
$K+3.6 \mu \mathrm{m}$ & 11 & 0.6 \\
$i+K$ & 33 & 1.9 \\
$i+3.6 \mu \mathrm{m}$ & 32 & 1.8 \\
$i$ & 15 & 0.8 \\
$K$ & 2 & 0.1 \\
$3.6 \mu \mathrm{m}$ & 6 & 0.3 \\
Unidentified & 10 & 0.6 \\
\hline
\end{tabular}

With the above definitions we have $~ 97 \%$ secure identifications, with $91 \%$ being secure in at least two bands.

The sources classified as ambiguous in the final identification are all sources that remain classified as such in both the $i$ and $K$ bands and in all the cases the same source was chosen as the primary solution in both bands.

Quite interestingly, there are 19 sources without an optical counterpart at the X-ray position (see Figure 5). Eleven of these have a $K$ and IRAC counterpart. Two sources have only a $K$-band counterpart, but one of them (CID-425) has an optical source visible in the HST image, where, however, the photometry has been totally contaminated by a bright nearby source. Six sources have only an IRAC counterpart.

Ten objects remain unidentified in all three bands. Only two of them are truly empty fields (CID-992, panel 7 in Figure 3, and CID-22962). The other eight sources belong to the unidentified class; however, a possible counterpart exists but is close to bright stars or galaxies, for which reliable photometry is not possible and there is not a entry in any COSMOS photometric catalog. Two examples (CID-734 and CID-561) are shown in panels 8 and 9 of Figure 3. These sources have been first associated by the likelihood method with the bright stars or galaxies nearby, which have been discarded after visual inspection. The X-ray centroid and contours are clearly pointing to the source missing from the catalog.

In summary, 20 sources do not have an optical counterpart listed in the catalog and also are truly empty fields in the optical. In X-ray-selected samples, nondetection in the optical band has often been assumed to be a proxy for high redshift, or for high obscuration level, or a combination of both, and in the past, such sources have been dubbed Extreme X-ray Objects (EXOs; e.g., Koekemoer et al. 2004). In this respect, only one of the sources with $K$ and IRAC identification has been spectroscopically observed (CID-472) and has been confirmed to be at high redshift $\left(z_{\text {spec }}=3.15\right)$. These optically unidentified sources have been included in the high-redshift space density computation presented in Civano et al. (2011).

The number of objects detected in all combinations of 3,2 , or 1 bands is reported in Table 2 . The sources with no detection in the optical should be the reddest objects $(R-K>5$, sources with $K$ and IRAC $3.6 \mu \mathrm{m}$, or only IRAC $3.6 \mu \mathrm{m}$ as in the examples shown in Figure 5). The objects detected in $i$ and IRAC bands but not in $K$ are in most cases close to bright sources $(K<18)$, so their photometry was not extracted in the $K$-band catalog (McCracken et al. 2010).

To estimate the total number of spurious associations in the field, the number of expected spurious associations among the secure sources (explained in Section 4.2) can be added to the number of sources with LR $<L_{\text {th }}$, many of which are indeed 


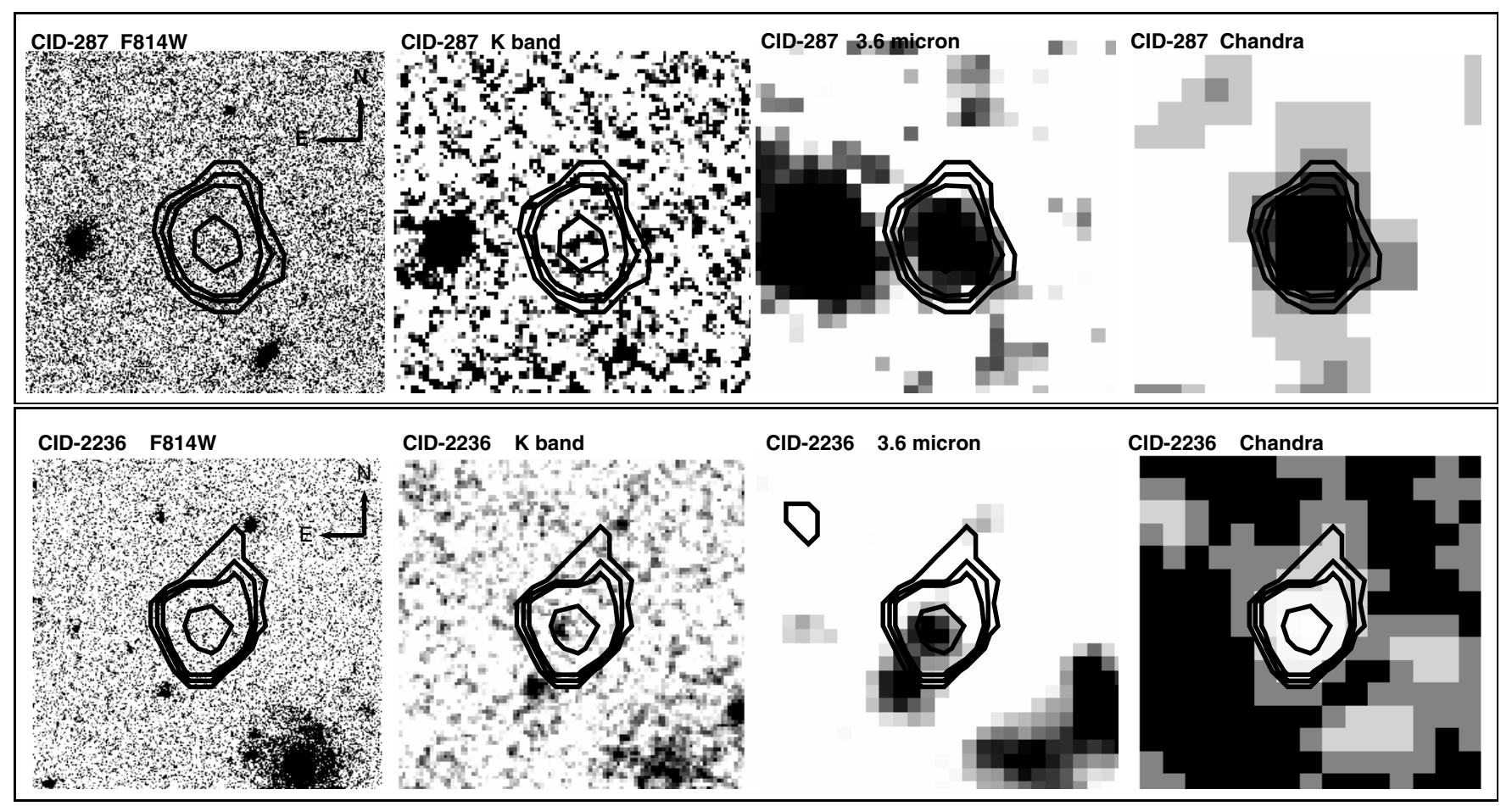

Figure 5. Examples of two sources (CID-287 and CID-2236) identified in the $K$ and $3.6 \mu \mathrm{m}$ bands but not in the $i$ band. ACS, $K, 3.6 \mu \mathrm{m}$, and $C h a n d r a$ cutouts are shown with the overlaid X-ray contours. Both sources are also very faint in the $K$ band.

expected to be either spurious associations or associated with spurious X-ray sources (see Section 5). However, since we used the combined information from the three bands and performed a visual inspection to isolate possible wrong matches, we expect that the final number of spurious associations will be lower. In conclusion. we can safely assume that it is $<6 \%$, which is in good agreement with the finding in the CDFS of Luo et al. (2010).

Finally, there are also seven X-ray sources (included in the secure sources) that are close to bright galaxies, for which the $\mathrm{X}$-ray contours are not centered on the galaxy itself but are clearly offset toward the outer region of the galaxy. These "offnuclear" sources have been discussed by Mainieri et al. (2010) and are candidates for ultraluminous X-ray sources.

\section{POSITIONAL OFFSETS AND MAGNITUDE DISTRIBUTIONS}

The distributions of the X-ray to $K, i$, and $3.6 \mu \mathrm{m}$ positional offsets are shown in Figure 6. The different colors of the histograms refer to the classes of identification (secure, black; ambiguous, red; subthreshold, green) as in the final classification (last column of Table 1). Almost $90 \%$ of the securely identified sources in each band lie within $1^{\prime \prime}$ of the X-ray centroids, which is consistent with the results obtained by other Chandra surveys (e.g., Brand et al. 2006; Laird et al. 2009; Green et al. 2009; Trichas et al. 2012; Luo et al. 2010; Xue et al. 2011; Goulding et al. 2012). This result is comparable to the XMM-COSMOS sample distance distribution (90\% within 3"; Brusa et al. 2007) taking into account the larger XMM-Newton PSF. If the most likely offset between an X-ray source and its counterpart is actually at $1 \sigma$ separation, according to the Rayleigh distribution, and given that the positional error ranges between 0.2 and 1".5 (from on-axis to off-axis sources; Figure 14 of Paper II) our results are in agreement with this expectation. The objects

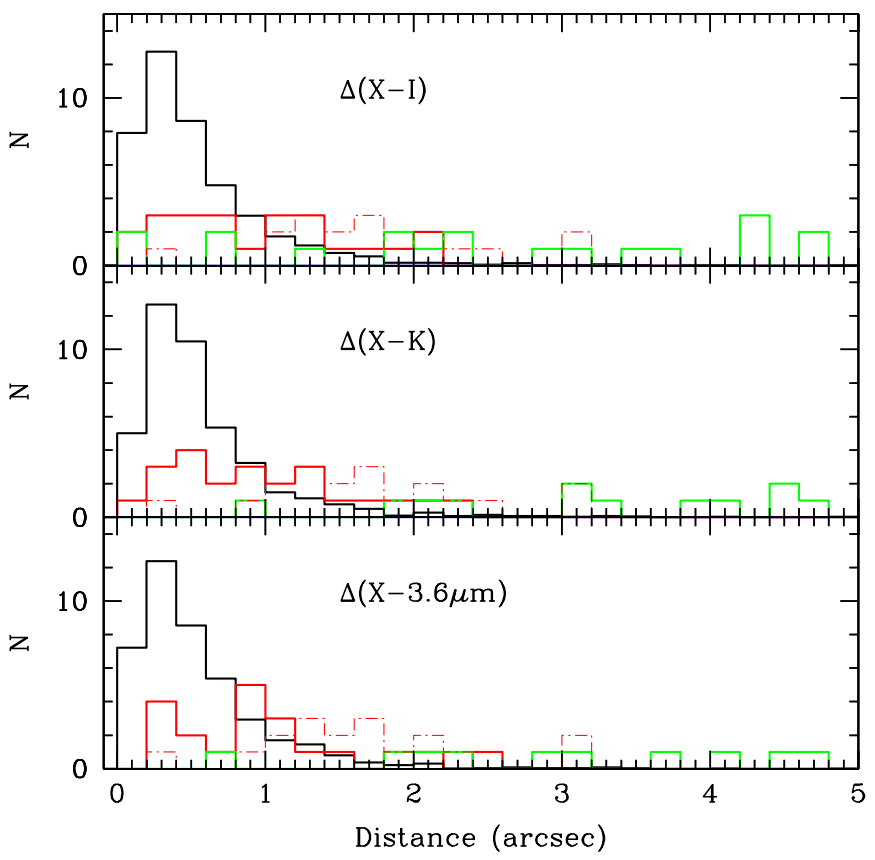

Figure 6. Histogram of the distances between the X-ray and $i$ (top), $K$ (middle), and IRAC $3.6 \mu \mathrm{m}$ (bottom) counterparts of the X-ray sources; black: secure sources (the histogram is divided by 40 for plotting purposes); red: ambiguous sources (thick: highest LR; dashed-thin: second possible); green: subthreshold sources; blue: faint retrieved sources (histogram is divided by 2 for plotting).

(A color version of this figure is available in the online journal.)

with offsets larger than 3".5 are mostly associated with low $\mathrm{S} / \mathrm{N}$ $\mathrm{X}$-ray sources (with less than 20 counts in the $0.5-8 \mathrm{keV}$ band) which have larger than average uncertainties on their X-ray position $\left(>1^{\prime \prime}\right)$. Two of them are associated with off-nuclear X-ray sources (Mainieri et al. 2010). 

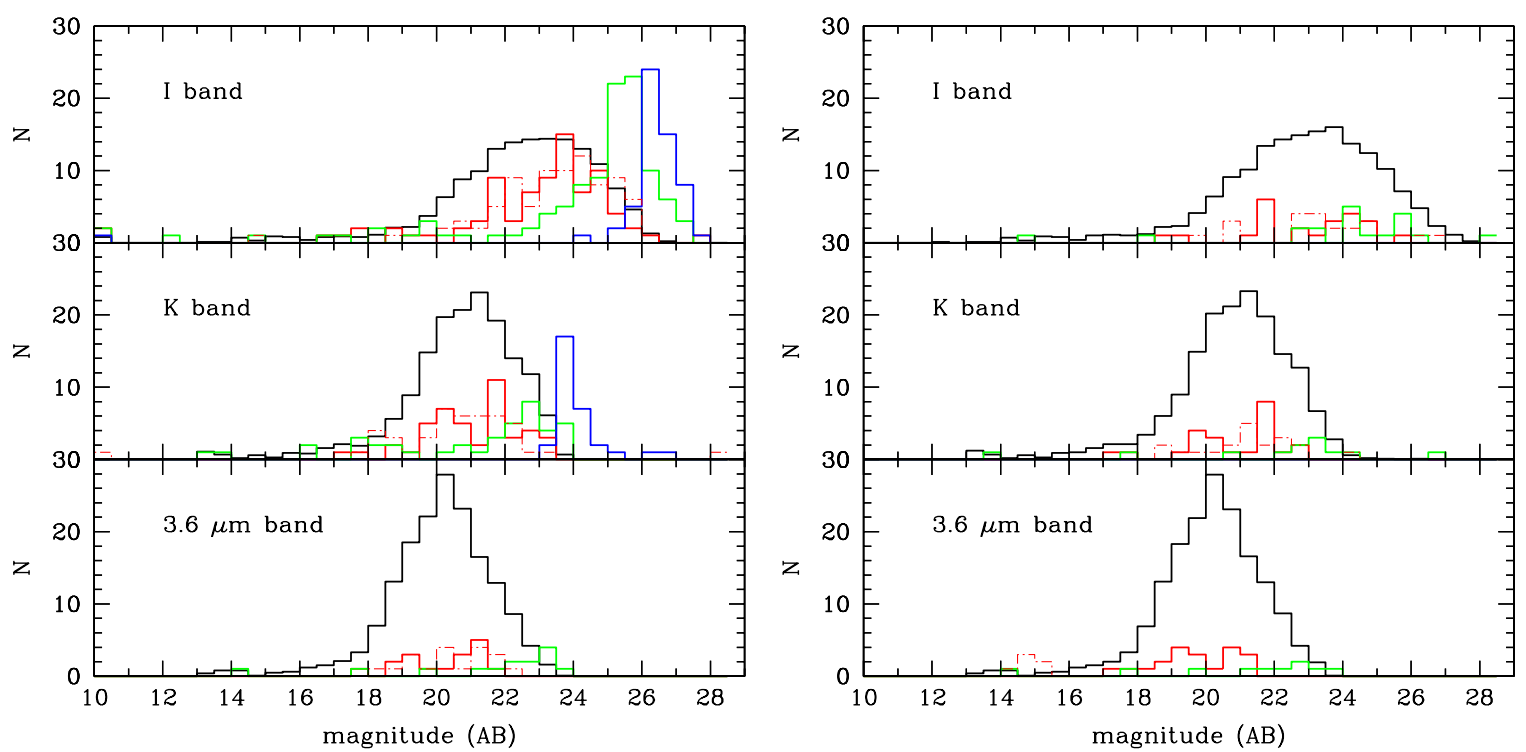

Figure 7. Distribution of the $i$ (top), $K$ (middle), $3.6 \mu \mathrm{m}$ (bottom) magnitudes. Black: secure sources (the histogram is divided by 10 for plotting purposes); red: ambiguous sources (thick: highest LR; dashed-thin: second possible); green: subthreshold sources; blue: faint retrieved sources. Left: the magnitude distribution according to the classification before the merging of the three bands, corresponding to Columns 4,6 , and 8 in Table 1 . Right: the magnitude distribution according to the final classification, after merging the results of the three bands, as in the last column of Table 1.

(A color version of this figure is available in the online journal.)

For ambiguous sources, the histograms of both the first (red solid line) and the second (red dashed line) possible counterparts are plotted. Both counterparts are close to the X-ray centroid (see panels 4-6 in Figure 3), but the first possible counterparts have a distribution that peaks at slightly lower offsets than the second. Only a few ambiguous sources with offsets larger than $2^{\prime \prime}$ are left after cross-correlating the three bands. The cross-correlation of the three catalogs reduces by half, with respect to the numbers in each band, the fraction of ambiguous sources in the final classification. The final percentage is only $2 \%$.

The histogram of offsets for retrieved counterparts (class (5), blue histogram) corresponding to Columns 4 and 6 of Table 1 has been reported only for comparison with the secure identification histograms. The blue histogram follows that of the securely identified counterparts, suggesting that they are as reliable as the brighter counterparts.

In Figure 7, the magnitude ${ }^{24}$ distributions of the $\mathrm{X}$-ray source counterparts in the $i$ (top), $K$ (middle), and $3.6 \mu \mathrm{m}$ (bottom) bands are shown before (left) and after (right) the merging of the classification in each band. Thus, in the left panel of Figure 7, we have five different classes (corresponding to Columns 4, 6, and 8 of Table 1), while in the right panel there are only four classes (corresponding to Column 9 of Table 1).

The histograms for both counterparts of the ambiguous sources (red solid and dot-dashed) follow the shape of the secure source histogram (black) and cover the same magnitude interval; their difference in LR is mainly due to the different positional offsets from the X-ray centroid. The primary counterpart (solid histograms in Figure 6) is closer to the X-ray position, while the secondary is slightly further away from the X-ray centroid.

The retrieved sources (blue histogram in Figure 7, left) lie within one magnitude of the limit of the histogram of the secure sources and, given that they do not show a significantly different distribution of X-ray to optical offsets, we consider them as being the fainter tail of the secure sources. Their contribution is

\footnotetext{
24 The magnitudes plotted are from aperture photometry: $3^{\prime \prime}$ in the optical, $3^{\prime \prime}$
} in the $K$ band, and 3". 8 in the IRAC band. visible in the final black histograms of the right panel of Figure 7. In contrast, the subthreshold objects (green histograms) show significantly larger X-ray to optical offsets and, on average, fainter magnitudes than the secure sources, so their low LR values are due to a combination of both factors. Six of the 19 subthreshold objects are detected in only one X-ray band with a low detection probability, and given the fact that 5,4 , and 3 spurious detections with more than 7 counts are expected in the full, soft, and hard bands, respectively, they could be associated with spurious X-ray sources.

\section{SPECTROSCOPIC AND PHOTOMETRIC REDSHIFT DISTRIBUTION}

The coordinates ${ }^{25}$ of each counterpart have been crosscorrelated with the spectroscopic catalogs currently available for the COSMOS field consisting of either data already present in the literature (SDSS DR7 $7^{26}$ and also Prescott et al. 2006) or data from the dedicated spectroscopic campaigns: the bright surveys, limited to $i<22.5$, with IMACS at Magellan, Hectospec at MMT (Trump et al. 2007, 2009a), and VIMOS at VLT (zCOSMOS bright; Lilly et al. 2007, 2009); the DEIMOS survey at Keck, limited to $i<25$, as the result of a multiyear observing campaign (PIs: Capak, Kartaltepe, Salvato, Sanders, Scoville; see Kartaltepe et al. 2010); the zCOSMOS deep survey to $B<25$ (Lilly et al. 2009); the star survey with the FAST spectrograph on the FLWO $1.5 \mathrm{~m}$ telescope (Wright et al. 2010). We also retrieved the WFC3 imaging and grism data of the COSMOS field (van Dokkum et al. 2011; Brammer et al. 2012) from the archive and processed them with standard software. The detection F140W image was reduced using the STSDAS pyraf task multidrizle (Koekemoer et al. 2003). The dispersed data (G141 grism) were reduced using the aXe slitless spectroscopy package Kümmel et al. (2009). The

\footnotetext{
${ }^{25}$ We used the optical coordinates for most sources, or the $K$-band coordinates if there is no optical counterpart, or $3.6 \mu \mathrm{m}$ coordinates if there is no $K$ or $i$ counterpart.

26 http://www.sdss.org/dr7/start/aboutdr7.html
} 
extracted two-dimensional and one-dimensional spectra were visually inspected and contaminated sources were rejected. From the set of 19 acceptable spectra, we determined new redshifts for 4 sources as well as confirmed 15 redshifts based on ground-based spectroscopy.

At present, 1069 objects $(60 \%)$ have spectroscopic redshifts (with one or more emission or absorption lines); $906(51 \%$ of the total) of these have a quality flag ( 3 or 4 , see Section 7) corresponding respectively to a secure redshift with two or more emission or absorption lines and a secure redshift with two or more emission or absorption lines with a good-quality, high $\mathrm{S} / \mathrm{N}$ spectrum (see Lilly et al. 2007, 2009 for thorough explanation of quality flags).

The available spectra are primarily the results of spectroscopic campaigns focused on XMM-COSMOS sources (Brusa et al. 2010). The spectroscopic campaigns targeting Chandraonly sources started recently, when the C-COSMOS counterparts were targeted by the deep zCOSMOS survey (Lilly et al. 2009) and by the DEIMOS survey. For these reasons, the fraction of sources that are not $X M M$-detected, yet have a spectroscopic redshift, is only $35 \%$. In the interim, photometric redshifts provide good estimates on the redshift range and classifications of the Chandra sources without a spectroscopic identification.

At a magnitude limit of $i=22.5$ (AB mag), C-COSMOS is $83 \%$ spectroscopically complete. If, at the same magnitude limit, we consider only bright X-ray fluxes similar to XMMCOSMOS ( $>10^{-15} \mathrm{erg} \mathrm{cm}^{-2} \mathrm{~s}^{-1}$ in the soft band), the survey is $89 \%$ spectroscopically complete, as most of the sources are in common with $X M M$, while, at lower X-ray fluxes $\left(<10^{-15} \mathrm{erg} \mathrm{cm}^{-2} \mathrm{~s}^{-1}\right)$, it is $76 \%$ complete. At fainter optical magnitudes $(>22.5)$, only $31 \%$ of sources have spectroscopic redshifts.

Tuned photometric redshifts for the C-COSMOS sources have been computed and presented in Salvato et al. (2011). In that paper, we used the publicly available code LePhare ${ }^{27}$ (Arnouts et al. 1999; Ilbert et al. 2006), which is based on a $\chi^{2}$ template-fitting procedure. Two libraries of templates were used, depending on a prior morphology, optical variability, and $\mathrm{X}$-ray flux of the source. The first library (defined in Salvato et al. 2009, Table 2) consists of AGN templates, hybrid (host + AGN) templates, and a few normal galaxies. It was used for all the point-like sources in the HST images (as defined by Leauthaud et al. 2007) that presented optical variability (see Salvato et al. 2011 for details), imposing a luminosity prior on the absolute $B$ magnitude typical for bright AGNs and QSOs $\left(-20<M_{B}<-30\right)$. The same library was also used for the extended sources with an X-ray flux brighter than $8 \times$ $10^{-15} \mathrm{erg} \mathrm{cm}^{-2} \mathrm{~s}^{-1}$. In this case, however, no luminosity prior was adopted. The second library (as defined in Ilbert et al. 2009) includes only normal galaxy templates and it was used for the remaining sources (i.e., extended, nonvariable, and with X-ray flux $<8 \times 10^{-15} \mathrm{erg} \mathrm{cm}^{-2} \mathrm{~s}^{-1}$ ), without any luminosity prior. The flowchart in Figure 6 of Salvato et al. (2011) summarizes the procedure.

In addition to the above two libraries, all the sources have also been fit by various stellar templates (low-mass stars, subdwarf $\mathrm{O}$ and $\mathrm{B}$ stars, white dwarfs $)$. Whenever $\left(2 \times \chi_{\text {star }}^{2}\right)<\chi_{\text {gal }}^{2}$, the source is assumed to be a star (see discussion in Salvato et al. 2011). For 10 sources not observed spectroscopically, the photometric fitting gives a better $\chi^{2}$ with a stellar template, and

\footnotetext{
27 www.oamp.fr/people/arnouts/LE_PHARE.html
}

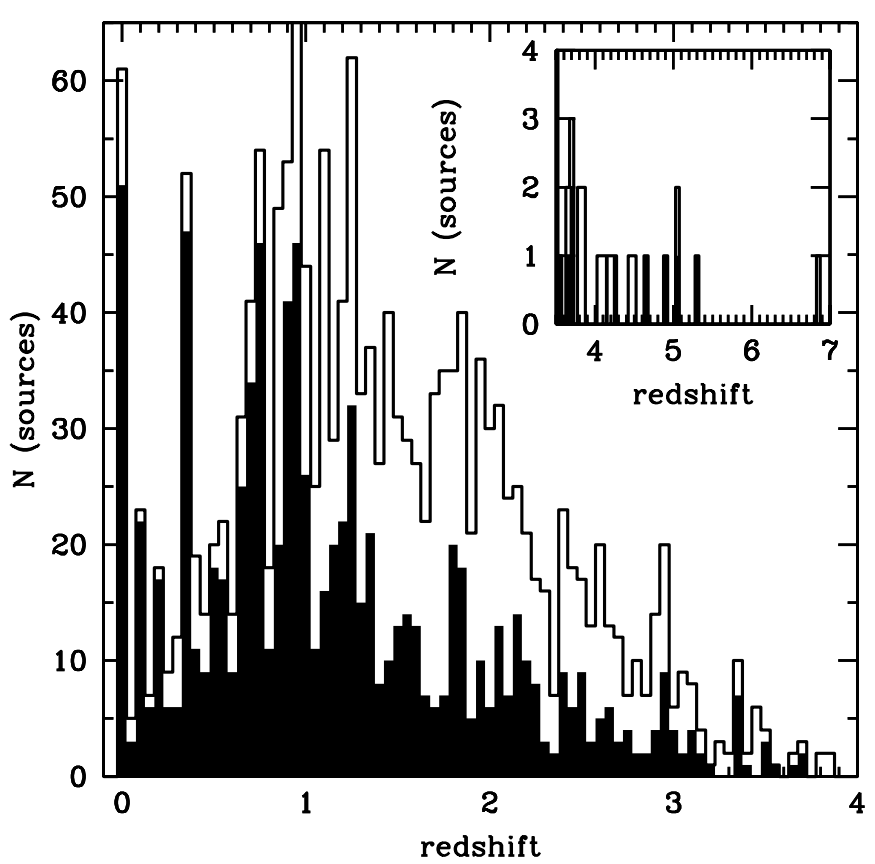

Figure 8. Redshift distribution of the optical counterparts $(\Delta z=0.05)$ - open histogram: all sources for which either a spectroscopic or photometric redshift is available; filled histogram: all sources for which a spectroscopic redshifts is measured (906). Inset: a zoom in the high- $z$ region $(z>3.5)$ has been plotted.

the visual inspection of the ACS images confirms the stellar nature of these sources.

The total number of sources with a photometric redshift is 1693. The accuracy achieved is $\sigma_{\Delta z} /\left(1+z_{\text {spec }}\right) \sim 1.5 \%$ (with $5.8 \%$ outliers) on the whole sample with no cut in magnitude or redshift applied. These results are comparable in precision to those typically achieved for nonactive galaxies (Cardamone et al. 2008; Wuyts et al. 2008; Ilbert et al. 2009; Barro et al. 2011) and to those reached only recently for photometric redshift of AGNs (Salvato et al. 2009; Cardamone et al. 2008; Luo et al. 2010; Fotopoulou et al. 2012) with a similar number of photometric bands.

The distribution of all the sources with a spectroscopic (906, solid histogram) or a photometric redshift (1693) is reported in Figure 8. The distribution peaks between $z=1-2$ and the spikes correspond to well-known large-scale structures in the COSMOS field (Gilli et al. 2009). There are 75 sources with redshift $>3$. These have been presented in Civano et al. (2011), where the number counts and the space density of the highredshift sample have been computed. While a sizable sample of high-redshift $z>5$ quasars has been collected in optical surveys (Fan et al. 2006; Willott et al. 2007), only two $z>5$ X-ray-selected AGNs have previously been reported in the literature: one in the CFDN (Steffen et al. 2004, $z=5.4$ ) and one in the CLASXS survey (Barger et al. 2005, $z=5.19$ ), both of them being unobscured type 1 sources. Given the faint optical magnitude, no sources with spectroscopic redshift at $z>5$ have been found in the CDFS, while there are five photometric candidates (Luo et al. 2010; Xue et al. 2011). In the C-COSMOS sample there are four sources at $z>5$, two of them with spectroscopic redshift (one with broad lines, Capak et al. 2011; one with narrow lines, Ikeda et al. 2011) and two with photometric redshift, one of them possibly being the highest redshift X-ray-selected source at $z=6.84$ (Salvato et al. 2011). 
Table 3

Number of X-Ray Sources by Spectral or Photometric Type

\begin{tabular}{|c|c|c|}
\hline & Number of Sources & $\%$ \\
\hline \multicolumn{3}{|c|}{ Spectra } \\
\hline Broad line & 316 & 35 \\
\hline Not broad line & 534 & 59 \\
\hline Star & 51 & 5.6 \\
\hline Off-nuclear (host galaxy) & 5 & 0.4 \\
\hline \multicolumn{3}{|c|}{ Photo- $z$} \\
\hline Unobscured AGN template & 450 & 27 \\
\hline Obscured AGN template & 104 & 6 \\
\hline Galaxy template & 1101 & 65 \\
\hline Star template & 30 & 2 \\
\hline Photometric star & 11 & \\
\hline Off-nuclear & 7 & \\
\hline
\end{tabular}

\subsection{Spectroscopic and Photometric Classification}

Table 3 breaks down the sources on the basis of their spectral type if spectra are available, or by the type of template that best fits the photometry of the sources.

Of the sources with good quality spectroscopic redshifts, $35 \%$ show at least one broad (FWHM $>2000 \mathrm{~km} \mathrm{~s}^{-1}$ ) emission line in their spectra (BLAGN), while 59\% do not present broad lines (i.e., they show narrow emission lines or absorption lines only), so we will refer to them as "nonbroad-line AGNs" (nonBLAGNs). The latter sources have not been classified into starforming galaxies or Type 2 AGNs because for most of them either the observed wavelength range or the low $\mathrm{S} / \mathrm{N}$ do not allow the use of standard optical diagnostic diagrams (e.g., Kewley et al. 2001). The remaining spectroscopic sources are spectroscopically identified stars (51 objects, $6 \%)$. The stars are mostly late-type $\mathrm{K}$ and $\mathrm{M}$ stars (35), with a number of solar-type $\mathrm{F}$ and $\mathrm{G}$ stars (16), that mostly lie at distances between 100 and $1000 \mathrm{pc}$, suggesting that they are primarily Galactic disk sources. A small fraction of these sources with high $\mathrm{X}$-ray luminosities lie at greater distances $(>1 \mathrm{kpc})$ and therefore are probably Galactic halo stars (Wright et al. 2010). Given the un-coherent spectroscopic follow-up, the percentages of the different spectral types are not necessarily representative of the whole C-COSMOS sample.

The classification obtained from the photometric fitting has been compared with the spectral classification for the sources with spectroscopic redshift. There is a good overall agreement between the two classifications: $81 \%$ of BLAGNs are fit with the template of an unobscured AGN, while $92 \%$ of the sources classified as non-BLAGNs are well fitted either by a galaxy template $(78 \%)$ or by an obscured AGN template $(22 \%)$. It is known that BLAGN SEDs, especially the low-luminosity ones, can be contaminated by stellar light (Luo et al. 2010; Lusso et al. 2010; Elvis et al. 2012); thus, it is not surprising that $19 \%$ of BLAGNs have a different classification than an unobscured source. Given this agreement, we can use either the spectroscopic or the photometric classification.

Sixty-five percent of the C-COSMOS sources are best fit by a nonactive galaxy, $6 \%$ by a template that represents an obscured AGN, and $27 \%$ by a template that includes an unobscured AGN component. The fraction of sources by type (both spectroscopic and photometric) as a function of the X-ray flux is plotted in Figure 9. The number of unobscured AGNs or BLAGNs decreases with the X-ray flux, while the fraction of galaxy SED sources or non-BLAGNs increases toward faint X-ray fluxes

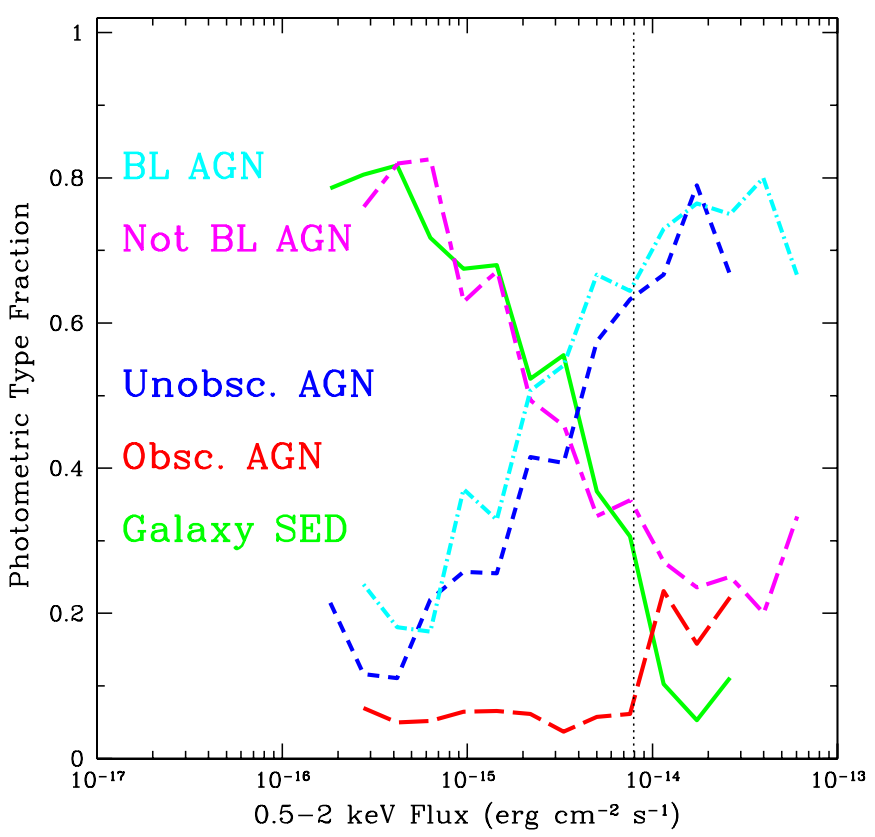

Figure 9. Fraction of the spectroscopic and photometric classification types of C-COSMOS opt/IR counterparts as a function of the soft-band X-ray flux. Cyan (short-long dashes): BLAGN; magenta (dot-dashed line): not BLAGN; blue (short dashes): unobscured AGN; red (long dashes): obscured AGN; green (solid): galaxies. The vertical dot-dashed line indicates the X-ray flux threshold below which, for extended and optically nonvarying sources, templates of normal galaxies have been used when computing photometric redshifts.

(A color version of this figure is available in the online journal.)

becoming the dominant population at fluxes fainter than $3 \times$ $10^{-15} \mathrm{erg} \mathrm{cm}^{-2} \mathrm{~s}^{-1}(0.5-2 \mathrm{keV})$. The galaxy SED source and obscured AGN fractions are complementary, because, as stated above, the optically extended C-COSMOS sources have been fitted with a galaxy template library if their X-ray flux is fainter than $8 \times 10^{-15} \mathrm{erg} \mathrm{cm}^{-2} \mathrm{~s}^{-1}(0.5-2 \mathrm{keV}$; vertical line in Figure 9) and with hybrid templates (obscured AGNs) if brighter. The small fraction of obscured AGNs at fluxes fainter than $8 \times$ $10^{-15} \mathrm{erg} \mathrm{cm}^{-2} \mathrm{~s}^{-1}$ (where all the extended sources have been fit only with galaxies templates) is due to the presence of sources with a point-like morphology, whose best fit is an obscured AGN.

A similar fraction for the galaxy SED objects (48\%) has been found in the photometric classification of CDFS sources at a flux limit of $1.9 \times 10^{-17} \mathrm{erg} \mathrm{cm}^{-2} \mathrm{~s}^{-1}(0.5-2 \mathrm{keV}$; Luo et al. 2010), while a lower fraction of unobscured AGNs is found (20\%) and a higher fraction of obscured AGNs (32\%). However, at the fluxes of the CDFS, $\sim 35 \%$ of the galaxy SED sources are likely to be low-luminosity normal and starburst galaxies (Xue et al. 2011; Lehmer et al. 2012).

In Figure 10, the X-ray luminosity versus redshift plane is shown for the soft (left) and hard (right) bands. To convert fluxes into luminosities, both spectroscopic and photometric redshifts were used (giving preference to the spectroscopic redshift, when available) and an X-ray spectral index $\Gamma=1.4$ (the same used to compute the X-ray fluxes in Paper I) was assumed to take into account the $k$-correction. In the figure we also report the faintest flux limits (dashed line) for XMM-COSMOS from Cappelluti et al. (2009, Table 2). There are 1323 and 1103 sources in the soft and hard bands, respectively, of which only $12 \%$ and $4 \%$ have luminosities possibly not due to nuclear emission or consistent with low-luminosity AGNs $\left(<10^{42} \mathrm{erg} \mathrm{s}^{-1}\right)$. The fact that only a small percentage of the sources has a luminosity consistent 

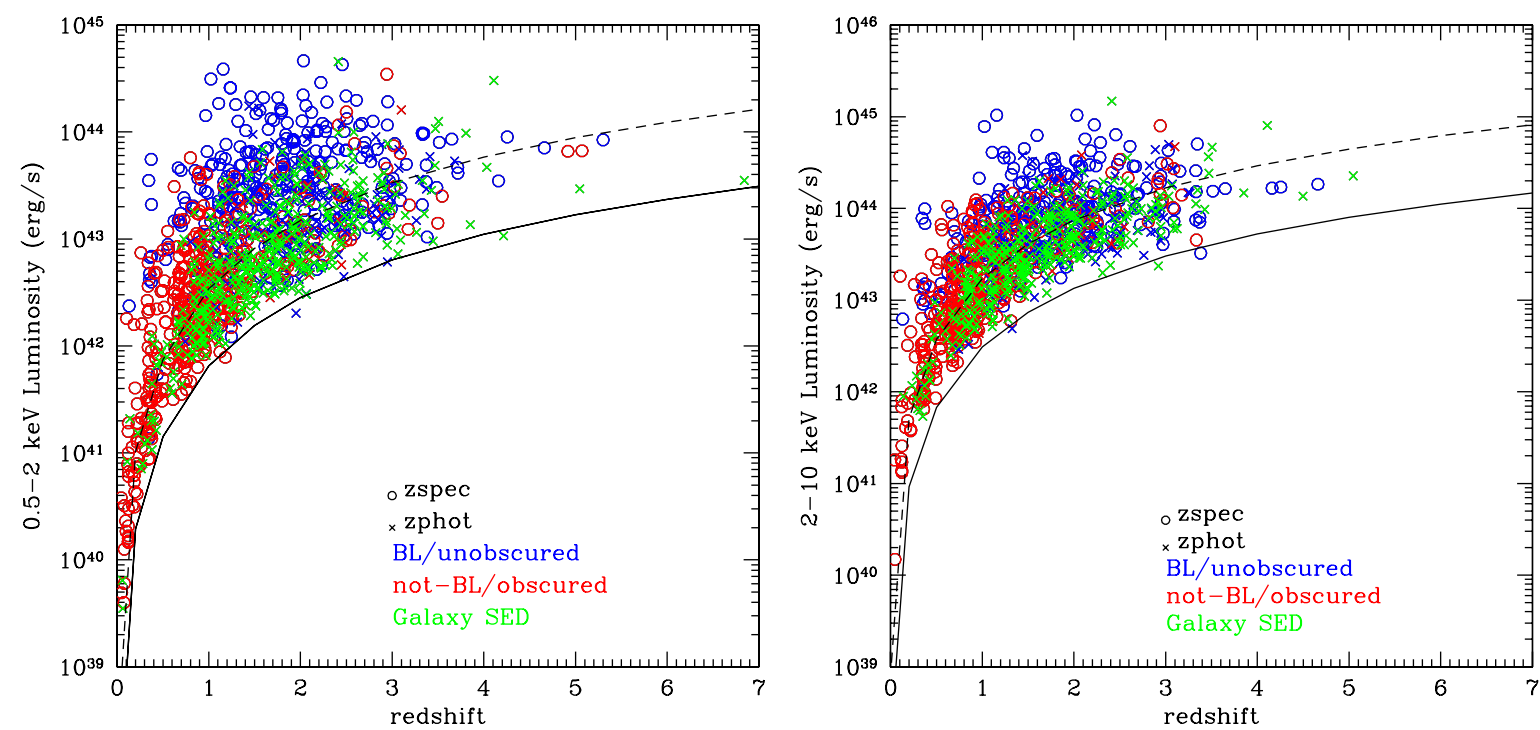

Figure 10. Luminosity-redshift plane (soft-left, hard-right) for all the sources with spectroscopic or photometric redshift, along with their classification. Sources with a spectroscopic identification are plotted as open circles, sources with only photometric identification as crosses; the symbols are color-coded as follows-blue: BLAGNs or unobscured AGNs from the SED; red: non-BLAGNs or obscured AGNs from the SED fitting; green: galaxy-dominated sources only from the SED. The solid lines represent the C-COSMOS flux limit and the dashed lines represent the XMM-COSMOS limit.

(A color version of this figure is available in the online journal.)

with that of a normal or starburst galaxy is in agreement with the fact that most of the galaxy SED sources (65\% of the total) are candidates to be obscured AGNs (as found in previous work, see Mainieri et al. 2005) and not normal galaxies.

In the rest of the paper we will use the $0.5-10 \mathrm{keV} \mathrm{lu}$ minosity to exclude normal and star-forming galaxies $\left(L_{X}<\right.$ $10^{42} \mathrm{erg} \mathrm{s}^{-1}$ ) from the sample. For 92 sources out of 906 with a spectroscopic redshift, a classification based on standard emission line diagnostic diagrams is available from Bongiorno et al. (2010): $66 \%$ of them (61 of 92) have emission line ratios typical of star-forming galaxies, while the remaining have the ratios of an obscured AGN. However, only $23 \%$ of those classified as star-forming galaxies (14 out of 61) have a luminosity $<10^{42} \mathrm{erg} \mathrm{s}^{-1}(0.5-10 \mathrm{keV})$, showing that optical diagnostic diagrams can be insensitive to hybrid objects (obscured AGNs with enhanced star formation). A luminosity cut at $10^{42} \mathrm{erg} \mathrm{s}^{-1}$ $(0.5-10 \mathrm{keV})$ may be a more reliable diagnostic to separate star-forming galaxies from AGNs, but at the risk of excluding bona fide, low-luminosity AGNs. The coupling of diagnostic diagrams with X-ray luminosities could then be a more effective method of separating obscured AGNs from truly nonactive galaxies (see also Trouille et al. 2011).

In the $1<z<2$ redshift bin, C-COSMOS luminosities span about two orders of magnitude $\left(10^{42.5}-10^{44.5} \mathrm{erg} \mathrm{s}^{-1}\right)$. $\mathrm{C}$-COSMOS covers the whole redshift range from 0 to 3 at $\mathrm{X}$-ray luminosities greater than $10^{43} \mathrm{erg} \mathrm{s}^{-1}$ in the soft band and $10^{43.5} \mathrm{erg} \mathrm{s}^{-1}$ in the hard band (solid lines in Figure 10). With this sample, it will be possible to derive accurate X-ray luminosity functions in both the hard and soft bands, in small redshift bins (T. Miyaji et al. 2012, in preparation).

\section{THE MULTIWAVELENGTH CATALOG OF C-COSMOS SOURCES}

The multiwavelength catalog of the Chandra COSMOS source identifications is available with the paper in the published version, in the COSMOS repository, ${ }^{28}$ and also at the following

\footnotetext{
28 http://irsa.ipac.caltech.edu/data/COSMOS/tables/
}

Web site. ${ }^{29}$ A sample of the catalog (truncated both in lines and columns) is reported in Table 4 . The catalog will contain the multiwavelength properties as listed below.

1. Column 1. Chandra source name, following the standard IAU convention with the prefix "CXOC" for "Chandra $X$-Ray Observatory COSMOS" survey, as in Paper I.

2. Column 2. Source number. Sources are listed in order of detection as in Paper I: first those detected in the full band with detml $\geqslant 10.8$, followed by those detected in the soft band only and by those detected in the hard band only.

3. Columns 3-4. The X-ray coordinates of the source from the Paper I catalog.

4. Columns 5-7. The X-ray flux in the full, soft, and hard bands. Negative fluxes represent upper limits as computed following the prescriptions of Paper II.

5. Columns 8-9. The coordinates of the optical/IR counterpart, as determined in this paper.

6. Column 10. The identifier number from the optical catalog of Capak et al. (2007).

7. Column 11. The identifier number from the revised version of the optical catalog (Ilbert et al. 2009).

8. Columns 12-13. The coordinates of the optical counterpart (Capak et al. 2007; Ilbert et al. 2009).

9. Columns 14-15. $i$-band magnitude and error in $3^{\prime \prime}$ aperture from the optical catalog.

10. Columns 16-17. $K$-band counterpart coordinates from the catalog of McCracken et al. (2010).

11. Columns 18-19. K-band aperture magnitude (at $3^{\prime \prime}$ ) and error from the catalog of McCracken et al. (2010).

12. Columns 20-21. The coordinates of the $3.6 \mu \mathrm{m}$ counterpart from the IRAC catalog (Sanders et al. 2007).

13. Columns 22-23.3.6 $\mu \mathrm{m}$ flux ( $\mu \mathrm{Jy}$ ) and error in 1".9 aperture from the IRAC catalog (Sanders et al. 2007). To convert to total flux, the standard factor suggested in the IRAC user guide has to be applied (division by 0.765 ).

\footnotetext{
${ }^{29}$ https://hea-www.cfa.harvard.edu/ fcivano/C_COSMOS
} identification_catalog.fits 
Table 4

Identification Catalog

\begin{tabular}{|c|c|c|c|c|c|c|c|c|c|c|c|c|c|c|}
\hline IAU Name & CID & X-Ray R.A. & X-Ray Decl. & $F_{0.5-2 \mathrm{keV}}$ & $F_{2-10 \mathrm{keV}}$ & $F_{0.5-10 \mathrm{keV}}$ & R.A. & Decl. & ID (Capak) & ID (Ilbert) & Optical R.A. & Optical Decl. & $\begin{array}{c}i \text {-band } \\
\text { Magnitude }\end{array}$ & $\begin{array}{c}i \text {-band } \\
\text { Magnitude Error }\end{array}$ \\
\hline CXOC 100040.9+021308 & 20 & 150.170460 & 2.218800 & $1.34 \mathrm{e}-15$ & $7.01 \mathrm{e}-15$ & $7.03 \mathrm{e}-15$ & 150.170470 & 2.218821 & 1617115 & 1004777 & 150.170470 & 2.218821 & 23.81 & 0.03 \\
\hline CXOC $100051.5+021216$ & 21 & 150.214640 & 2.204334 & $6.82 \mathrm{e}-15$ & $1.60 \mathrm{e}-14$ & $2.59 \mathrm{e}-14$ & 150.214660 & 2.204285 & 1620425 & 1007423 & 150.214660 & 2.204285 & 21.05 & 0.02 \\
\hline CXOC $100049.5+021709$ & 22 & 150.206260 & 2.285839 & $6.73 e-15$ & $1.89 \mathrm{e}-14$ & $2.54 \mathrm{e}-14$ & 150.206270 & 2.285746 & 1600414 & 994808 & 150.206270 & 2.285746 & 22.59 & 0.02 \\
\hline CXOC $100053.7+021615$ & 23 & 150.223910 & 2.270800 & $1.41 \mathrm{e}-15$ & $4.39 \mathrm{e}-15$ & $5.45 \mathrm{e}-15$ & 150.224030 & 2.270799 & 1604459 & 997226 & 150.224030 & 2.270799 & 23.86 & 0.04 \\
\hline CXOC 100054.7+021611 & 24 & 150.227990 & 2.269833 & $1.34 \mathrm{e}-15$ & $5.24 \mathrm{e}-15$ & $5.83 \mathrm{e}-15$ & 150.228090 & 2.269819 & 1565459 & 971505 & 150.228090 & 2.269819 & 21.17 & 0.01 \\
\hline CXOC $100055.3+021520$ & 25 & 150.230570 & 2.255563 & $6.60 \mathrm{e}-16$ & $2.04 \mathrm{e}-15$ & $2.85 \mathrm{e}-15$ & 150.230670 & 2.255365 & 1569619 & 974083 & 150.230670 & 2.255365 & 25.06 & 0.08 \\
\hline CXOC 100056.7+021721 & 26 & 150.236200 & 2.289173 & $2.14 \mathrm{e}-15$ & $2.82 \mathrm{e}-15$ & $6.48 \mathrm{e}-15$ & 150.236250 & 2.289117 & 1561491 & 969546 & 150.236250 & 2.289117 & 21.51 & 0.03 \\
\hline CXOC 100100.0+021253 & 28 & 150.250170 & 2.214593 & $6.67 \mathrm{e}-16$ & $1.35 \mathrm{e}-14$ & $8.59 \mathrm{e}-15$ & 150.250210 & 2.214590 & 1578009 & 979686 & 150.250210 & 2.214590 & 20.41 & 0.01 \\
\hline CXOC $100100.5+021543$ & 29 & 150.252280 & 2.261923 & $2.42 \mathrm{e}-15$ & $2.83 \mathrm{e}-15$ & $7.92 \mathrm{e}-15$ & 150.252240 & 2.261908 & 1567840 & 972975 & 150.252240 & 2.261908 & 23.25 & 0.03 \\
\hline CXOC 100105.3+021348 & 31 & 150.272070 & 2.230126 & $7.71 \mathrm{e}-15$ & $1.85 \mathrm{e}-14$ & $2.99 \mathrm{e}-14$ & 150.272140 & 2.230096 & 1574366 & 978155 & 150.272140 & 2.230096 & 20.80 & 0.02 \\
\hline CXOC 100109.9+021728 & 32 & 150.291220 & 2.291028 & $1.54 \mathrm{e}-15$ & $1.56 \mathrm{e}-15$ & $4.83 \mathrm{e}-15$ & 150.291250 & 2.290990 & 1561180 & 969205 & 150.291250 & 2.290990 & 23.41 & 0.03 \\
\hline CXOC 100111.0+021634 & 33 & 150.296040 & 2.276037 & $1.74 \mathrm{e}-15$ & $3.28 \mathrm{e}-15$ & $6.04 \mathrm{e}-15$ & 150.296030 & 2.276179 & 1564589 & 970989 & 150.296030 & 2.276179 & 23.62 & 0.03 \\
\hline CXOC 100112.4+021656 & 34 & 150.301550 & 2.282217 & $2.28 \mathrm{e}-15$ & $4.07 e-15$ & $7.68 \mathrm{e}-15$ & 150.301580 & 2.282146 & 1563682 & 970356 & 150.301580 & 2.282146 & 26.21 & 0.18 \\
\hline CXOC 100046.7+020405 & 35 & 150.194690 & 2.067936 & $8.41 \mathrm{e}-15$ & $1.12 \mathrm{e}-14$ & $2.67 e-14$ & 150.194710 & 2.067906 & 1247183 & 773587 & 150.194710 & 2.067906 & 20.17 & 0.01 \\
\hline CXOC 100038.0+020822 & 36 & 150.158320 & 2.139531 & $4.00 \mathrm{e}-15$ & $8.08 \mathrm{e}-15$ & $1.40 \mathrm{e}-14$ & 150.158380 & 2.139603 & 1636953 & 1017264 & 150.158380 & 2.139603 & 20.40 & 0.01 \\
\hline CXOC 100109.1+020755 & 37 & 150.287920 & 2.131910 & $3.76 \mathrm{e}-15$ & $6.31 \mathrm{e}-15$ & $1.25 \mathrm{e}-14$ & 150.288000 & 2.131841 & 1597415 & 992777 & 150.288000 & 2.131841 & 22.60 & 0.02 \\
\hline CXOC $100102.8+020317$ & 38 & 150.261660 & 2.054727 & $2.33 \mathrm{e}-15$ & $9.06 \mathrm{e}-15$ & $1.03 \mathrm{e}-14$ & 150.261780 & 2.054619 & 1213002 & 751616 & 150.261780 & 2.054619 & 22.71 & 0.02 \\
\hline CXOC 100104.2+020320 & 39 & 150.267510 & 2.055629 & $2.91 \mathrm{e}-15$ & $7.51 \mathrm{e}-15$ & $1.09 \mathrm{e}-14$ & 150.267440 & 2.055690 & 1212775 & 751114 & 150.267440 & 2.055690 & 24.71 & 0.06 \\
\hline CXOC 100047.9+021127 & 40 & 150.199740 & 2.190907 & $4.69 \mathrm{e}-15$ & $9.69 \mathrm{e}-15$ & $1.63 \mathrm{e}-14$ & 150.199750 & 2.190866 & 1623780 & 1009041 & 150.199750 & 2.190866 & 21.08 & 0.02 \\
\hline CXOC $100040.9+020717$ & 41 & 150.170400 & 2.121475 & $1.31 \mathrm{e}-15$ & $4.22 \mathrm{e}-15$ & $4.81 \mathrm{e}-15$ & 150.170220 & 2.121434 & 1235583 & 766637 & 150.170220 & 2.121434 & 24.63 & 0.06 \\
\hline CXOC $100043.2+020637$ & 42 & 150.179800 & 2.110340 & $1.83 \mathrm{e}-14$ & $3.62 \mathrm{e}-14$ & $6.47 \mathrm{e}-14$ & 150.179790 & 2.110378 & 1236435 & 767213 & 150.179790 & 2.110378 & 19.17 & 0.01 \\
\hline CXOC 100045.5+020935 & 44 & 150.189660 & 2.159718 & $1.23 \mathrm{e}-15$ & $3.56 \mathrm{e}-15$ & $5.23 \mathrm{e}-15$ & 150.189580 & 2.159761 & 1631490 & 1014432 & 150.189580 & 2.159761 & 23.86 & 0.04 \\
\hline CXOC 100046.6+020625 & 45 & 150.194260 & 2.106866 & $6.44 \mathrm{e}-16$ & $5.88 \mathrm{e}-15$ & $5.32 \mathrm{e}-15$ & 150.194280 & 2.106767 & 1239109 & 768974 & 150.194280 & 2.106767 & 24.41 & 0.05 \\
\hline CXOC 100047.0+020710 & 46 & 150.195810 & 2.119341 & $1.38 \mathrm{e}-15$ & $6.99 \mathrm{e}-15$ & $7.13 e-15$ & 150.196080 & 2.119272 & 1236440 & 766977 & 150.196080 & 2.119272 & 23.82 & 0.04 \\
\hline CXOC 100047.7+020757 & 47 & 150.198900 & 2.132551 & $9.66 \mathrm{e}-16$ & $6.00 \mathrm{e}-15$ & $6.09 \mathrm{e}-15$ & 150.198970 & 2.132537 & 1637411 & 1018336 & 150.198970 & 2.132537 & 19.66 & 0.01 \\
\hline
\end{tabular}

Note. (FITS files of this table available in the online journal.) 

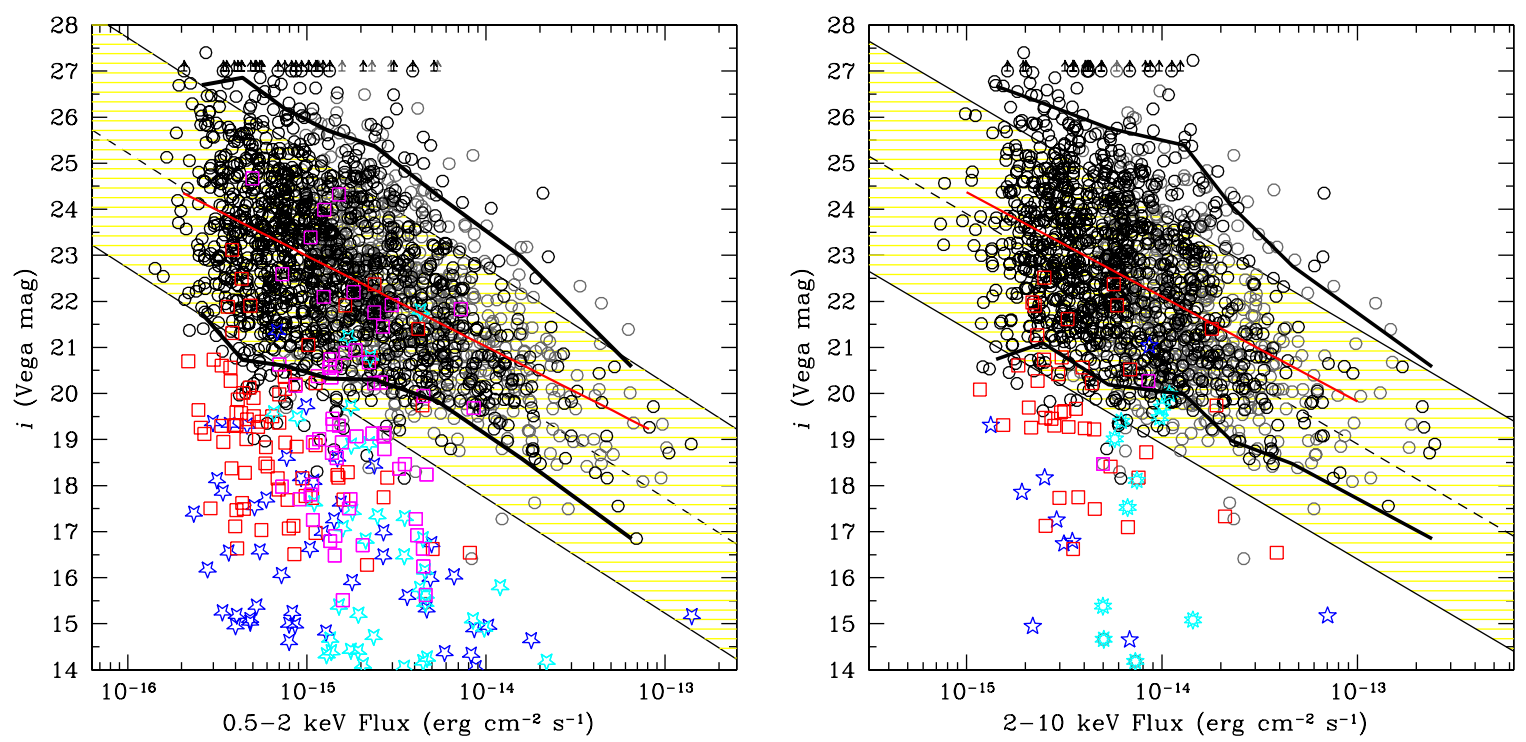

Figure 11. X-ray flux (soft-left, hard-right) vs. the $i$-band total (i.e., aperture corrected) magnitude for all the X-ray sources with an $i$-band counterpart. The yellow shaded region represents the "classic locus" and the "hard-band locus" of AGNs along the correlation X/O $=0 \pm 1$. Black symbols: C-COSMOS sources. Gray symbols: XMM-COSMOS sources. Star symbols (blue: Chandra; cyan: XMM) represent spectroscopic or photometric identified stars. Red and magenta squares represent sources with $L_{F}<10^{42} \mathrm{erg} \mathrm{s}^{-1}$ in C-COSMOS and XMM-COSMOS, respectively. Sources not identified in the optical but with a counterpart in the $K$ or IRAC band are represented with upward arrows. The thick solid curves represent the newly drawn locus. The red solid line is the fit to the combined (Chandra and $X M M)$ sample, respectively.

(A color version of this figure is available in the online journal.)

14. Column 24. Final identification flag: $1=$ secure, $10=$ ambiguous, $-99=$ unidentified, $100=$ subthreshold.

15. Column 25. Star flag to isolate stars: $1=$ spectroscopic confirmed star, $10=$ photometric star, $100=$ visually identified star.

16. Column 26. Off-nuclear flag to isolate the seven off-nuclear sources from Mainieri et al. (2010).

17. Column 27. Spectroscopic redshift from the catalogs listed above (Section 6).

18. Column 28. Spectroscopic identification. The identification flag can be read as follows: $1=$ BLAGN, $2=$ non-BLAGN, $0=$ star.

19. Column 29. Spectroscopic redshift quality: $3=$ two or more emission and/or absorption lines, $4=$ good signal-to-noise ratio and two or more emission and/or absorption lines.

20. Column 30. Origin of the spectroscopic redshift: $1=$ SDSS, $2=\mathrm{MMT}, 3$ or $4=\mathrm{IMACS}, 5=\mathrm{zCOSMOS}$ bright, $6=$ zCOSMOS deep, $7=$ Keck $8=$ other (FAST telescope, FMOS, WFC3).

21. Column 31. Photometric redshift from Salvato et al. (2011).

22. Column 32. Photometric identification from the SED fitting $(1=$ unobscured, $2=$ obscured, $3=$ galaxy $)$.

23. Column 33. The identifier number in the XMM-COSMOS catalog (Cappelluti et al. 2009).

\section{OPTICAL AND INFRARED PROPERTIES}

In this section the basic X-ray to optical properties of the sample are reported. Hereafter, we will consider only the 1708 sources with secure identifications and the counterpart with the highest LR for the 24 sources with ambiguous associations. We will not consider the 7 off-nuclear sources (Mainieri et al. 2010), the 62 stars (Wright et al. 2010), the 10 unidentified sources, and the 19 subthreshold sources.

\subsection{X-Ray to Optical Flux Ratio}

The X/O ratio (Maccacaro et al. 1988) is defined as

$$
\mathrm{X} / \mathrm{O}=\log \left(f_{X} / f_{\mathrm{opt}}\right)=\log \left(f_{X}\right)+C+m_{\mathrm{opt}} / 2.5,
$$

where $f_{X}$ is the X-ray flux in a given energy range, $m_{\text {opt }}$ is the magnitude at the chosen optical wavelength, and $C$ is a constant which depends on the specific filter used in the optical observations. Usually, the $r$ - or $i$-band flux is used (e.g., Brandt \& Hasinger 2005). Originally, a soft X-ray flux was used for this relation (see the Introduction), and the majority of luminous spectroscopically identified AGNs (both BLAGNs and NLAGNs) in the Einstein and ASCA surveys were characterized by $\mathrm{X} / \mathrm{O}=0 \pm 1$ (Schmidt et al. 1998; Stocke et al. 1991; Akiyama et al. 2000; Lehmann et al. 2001). With the advent of harder surveys, the same relation has been used in the hard band, without really accounting for the X-ray band used or the change in spectral slope (Alexander et al. 2001; Hornschemeier et al. 2001; Fiore et al. 2003; Brusa et al. 2003, 2007; Perola et al. 2004; Civano et al. 2005; Cocchia et al. 2007; Laird et al. 2009; Xue et al. 2011).

In Figure 11, the $i$-band total magnitude is plotted versus the X-ray soft (left) and hard (right) fluxes for all the sources with a secure identification. For both bands, the $\mathrm{X} / \mathrm{O}= \pm 1$ locus (yellow area) has been defined using as $C(i)=5.91$ the constant, which was computed taking into account the width of the $i$-band filters in COSMOS (Subaru, CFHT, or for bright sources SDSS). In the hard band, the locus (hereafter the "hardband locus") is plotted taking into account the band width and the spectral slope used to compute the C-COSMOS fluxes $(\Gamma=1.4)$. The difference between the "classic locus" and the "hard-band locus" is X/O $=+0.47$. Sources not identified in the optical, but with a counterpart in the $K$ or IRAC $3.6 \mu \mathrm{m}$ band, are represented with upward arrows at $i=27$. The stars in the sample are represented with blue star symbols and sources with 

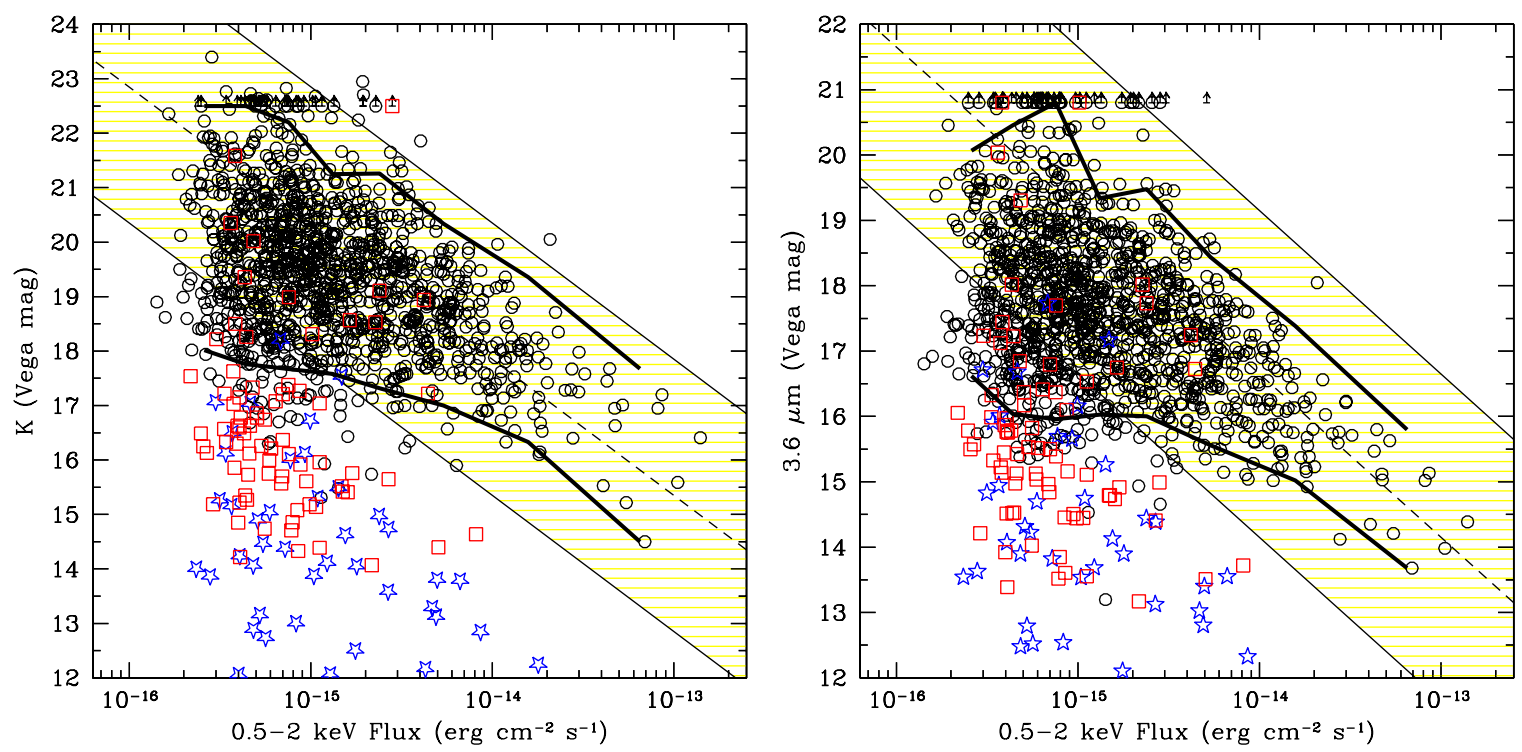

Figure 12. X-ray soft flux vs. the $K$ (left) and $3.6 \mu \mathrm{m}$ (right) total (i.e., aperture corrected) magnitudes for all the X-ray sources. The yellow shaded region represents the "classic locus" of AGNs along the correlation $\mathrm{X} / \mathrm{O}=0 \pm 1$. Black symbols: C-COSMOS sources. Star symbols represent spectroscopic or photometric identified stars. Red and magenta squares represent sources with $L_{F}<10^{42} \mathrm{erg} \mathrm{s}^{-1}$ in C-COSMOS and XMM-COSMOS, respectively. Sources not identified in these bands are represented with upward arrows. The thick solid curves represent the region including $90 \%$ of the AGN population.

(A color version of this figure is available in the online journal.)

a full-band luminosity $L_{F}<10^{42} \mathrm{erg} \mathrm{s}^{-1}$ are represented with red squares.

The X-ray versus optical flux plot is quite homogeneously covered by the C-COSMOS sources with a large number of sources outside the $\mathrm{X} / \mathrm{O}=0 \pm 1$ locus, mostly in the hard band (Figure 11, right). In order to quantify the width of the distribution (i.e., the $\mathrm{X} / \mathrm{O}$ distributions), we computed the region which includes $90 \%$ of the AGN population (i.e., excluding sources identified as stars and sources with $L_{F}<10^{42} \mathrm{erg} \mathrm{s}^{-1}$ ) by tracing the 5\% lower percentile and $95 \%$ upper percentile of the distributions of $i$-band magnitudes for sources in $\mathrm{X}$-ray flux bins of width 0.25 dex (thick black solid curves in Figure 11). In deriving the $95 \%$ upper percentile, we included the non-detections in the optical band. A total of 1274 and 1115 sources were used in the soft and hard bands, respectively. In the following we refer to this region as the newly drawn locus.

In the soft band, the newly drawn locus fits the "classic locus" of AGNs at bright fluxes very well but widens at fainter fluxes, shifting to faint optical magnitudes by $\Delta(\mathrm{X} / \mathrm{O}) \sim 0.5$. In the hard band, the newly drawn locus behaves similarly to the one in the soft band with the same shift of $\Delta(\mathrm{X} / \mathrm{O}) \sim 0.5$ to faint optical magnitudes. This shift is consistent with the $\mathrm{X} / \mathrm{O}$ relation being originally calibrated on soft-X-ray-selected sources, bright in the optical and also in the X-rays. The width of the locus in both bands is constant over 1.5 dex. The population of sources outside the newly drawn locus is constant in number in the soft band while it becomes larger (in number) at fainter fluxes in the hard band.

We also performed a linear regression fitting ${ }^{30}$ to the logarithm of the X-ray flux and the total (aperture corrected) $i$-band magnitude. In order to have better statistics at bright fluxes, we also included the XMM-COSMOS sources (outside the Chan$d r a$ area) in the fit using the catalog of identifications by Brusa et al. (2010). The advantage of including the XMM-COSMOS sources is that the optical photometric catalog is the same, so problems of calibration using different filters are not an issue.

\footnotetext{
30 The ROBUST_LINEFIT IDL routine is used.
}

Moreover, the good calibration between the Chandra and XMMNewton fluxes (5\%-10\%; G. Lanzuisi et al., in preparation, hereafter Paper IV) should not affect the result. Adding the XMM-COSMOS sources creates a sample of 1921 AGNs in the soft band and 1597 AGNs in the hard band. In Figure 11 we show the fit in both bands computed using the combined sample (red solid line). The fit is consistent with the newly drawn locus using the median optical magnitude in flux bins. The Spearman rank correlation coefficient of the fit to the combined sample is $\rho=-0.52$ for the soft band and $\rho=-0.48$ for the hard band, with a significance level $>99.9 \%$. The linear relations found for the two bands are $i_{\mathrm{AB}}=(-5.88 \pm 1.02)+(-1.9 \pm 0.1) \times \log F_{X}$ (soft) and $i_{\mathrm{AB}}=(-9.68 \pm 1.39)+(-2.2 \pm 0.1) \times \log F_{X}$ (hard).

In Figure 12 we show the X-ray soft flux versus the total $K$ (left) and $3.6 \mu \mathrm{m}$ (right) magnitudes for all the X-ray sources as in Figure 11. The "classic locus," obtained using Equation (3) and the constant $C=6.86$ and 7.34 for the $K$ and $3.6 \mu \mathrm{m}$, respectively, is plotted in the figure, as well as the region that includes $90 \%$ of the AGN population, as computed for the $i$ band. Similar plots have been shown before in the $K$ band by Mainieri et al. (2002) and Brusa et al. (2005) and at $3.6 \mu \mathrm{m}$ by Xue et al. (2011) and Laird et al. (2009). In both bands, the spread of the AGN population is reduced by $1.2-2$ mag with respect to the $i$ band. This suggests a stronger coupling between the X-ray flux and the near-infrared wavelengths than in the $i$ band. The higher identification rates in these bands had already hinted at this connection. The reduced spread in the near-infrared bands is mainly due to the reduced nuclear extinction which, on the contrary, strongly affects the bluer bands.

\subsubsection{X-Ray to Optical Flux Ratio and Photometric Classification}

We also used the photometric classifications available for a large fraction of the sources via SED fitting to compute the median $\mathrm{X} / \mathrm{O}$, dividing the sources into three classes (unobscured AGNs, obscured AGNs, and galaxy SED sources). This allows us to investigate how the $\mathrm{X} / \mathrm{O}$ distributions change with $\mathrm{X}$-ray band and source classification. The median values of $\mathrm{X} / \mathrm{O}$ in the 
Table 5

X/O Median Values and $68 \%$ and $90 \%$ Ranges in the Soft and Hard Bands for Each SED Type

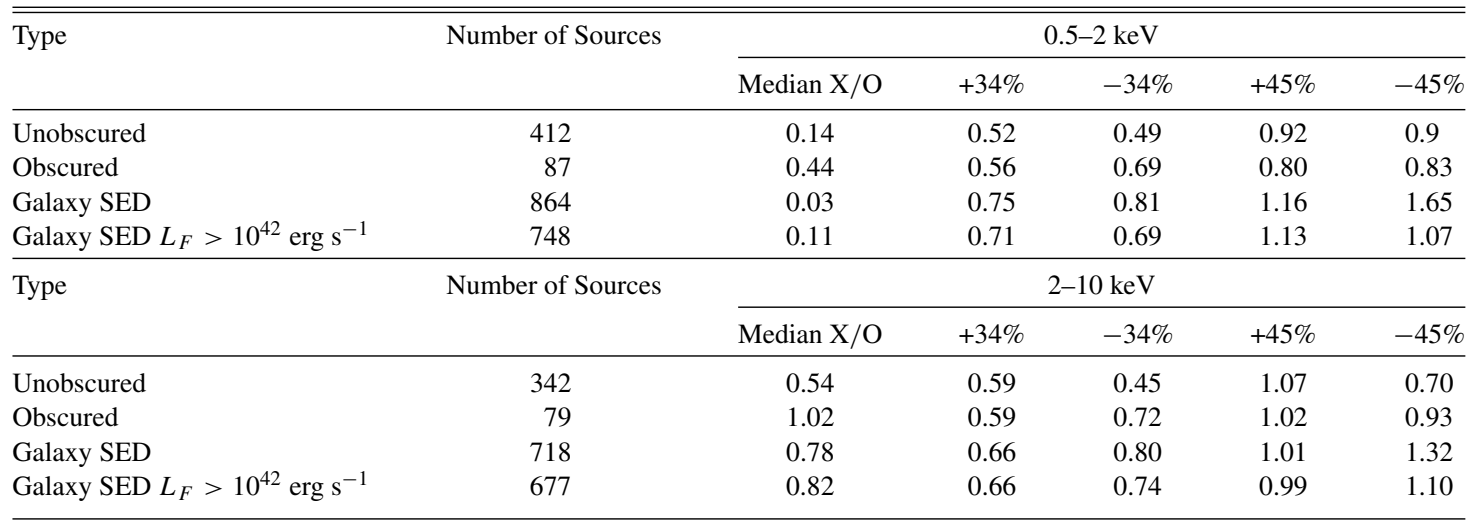

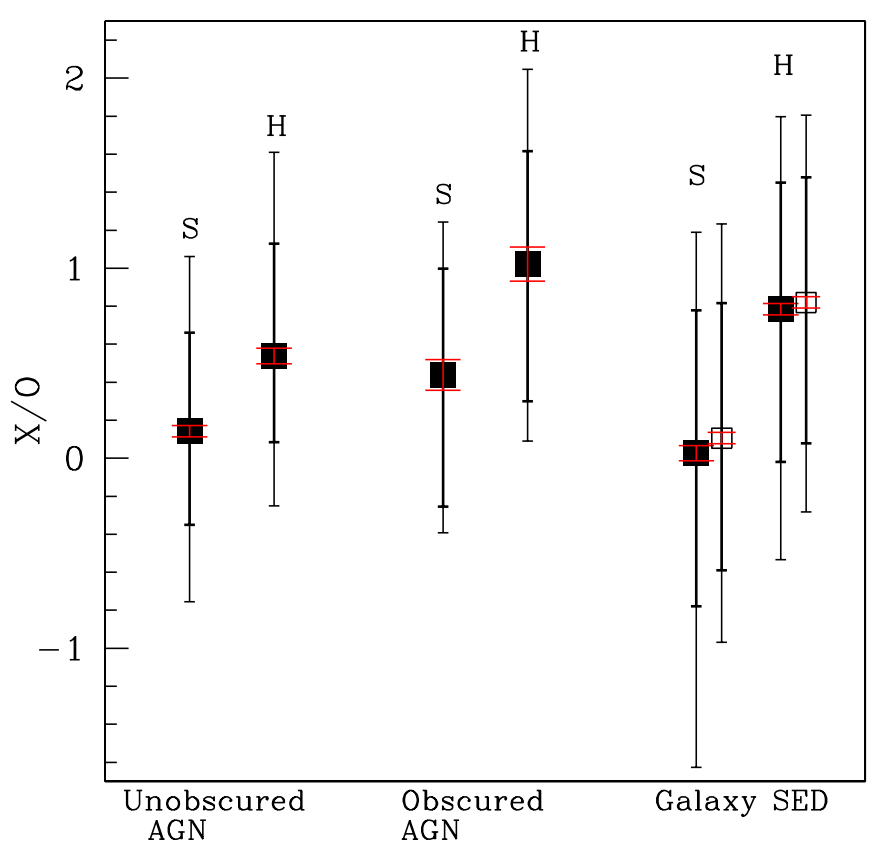

Figure 13. Median X-ray to optical flux ratio in the soft $(\mathrm{S})$ and hard $(\mathrm{H})$ bands for the sources divided in SED classes (unobscured AGNs, obscured AGNs, and galaxy SED sources). The open squares represent galaxy SED sources with $L_{F}>10^{42} \mathrm{erg} \mathrm{s}^{-1}$. The black bars represent the $68 \%$ and $90 \%$ dispersion. The red error bars represent the median deviation. A table with median values and $68 \%$ and $90 \%$ dispersion is available in Table 5.

(A color version of this figure is available in the online journal.)

hard and soft bands for each class along with the $68 \%$ and $90 \%$ ranges are reported in Figure 13 and in Table 5. The unobscured AGNs fit the "classic locus" $(-1<\mathrm{X} / \mathrm{O}<1$ in the soft) and the "hard-band locus" $(-0.53<\mathrm{X} / \mathrm{O}<1.47)$ while the obscured AGNs have a higher X/O than unobscured AGNs and the difference is larger in the hard band $(\sim 0.5 \mathrm{dex})$ than in the soft band $(\sim 0.3$ dex $)$. Their difference in $\mathrm{X} / \mathrm{O}$ distributions is also confirmed by a Kolmogorov-Smirnov (K-S; $P=0.0006)$. The $90 \%$ range of obscured AGNs spreads to $\mathrm{X} / \mathrm{O}>2$.

The X/O distribution of galaxy SED sources spans a very large range. In the soft band, the spread at low $\mathrm{X} / \mathrm{O}$ values is due to the presence of low-luminosity normal galaxies (red squares in Figure 11), and it decreases when only luminous sources are considered (open squares in Figure 13). In the hard band, the galaxy SED sources have a median X/O closer to that of obscured AGNs and with a smaller spread to low X/O than in the soft band because the contribution of normal galaxies to the hard-band $\mathrm{X} / \mathrm{O}$ is negligible (as shown in Figure 11). A K-S test confirms that obscured AGNs and galaxy SED sources are consistent with being drawn from the same parent population ( $P=0.46$ and $P=0.58$ when only bright galaxy SED sources are included).

As has already been argued and observed by several authors, obscured accretion at high redshift and the fact that the optical flux is more affected by absorption than the X-ray flux are responsible for high X-ray to optical flux ratios. When redshifting the SED of an obscured AGN to progressively higher redshifts, the $k$-corrections in the optical and X-ray band work in opposite directions. The X-ray flux, with a typical extremely hard spectral slope, has a positive $k$-correction, increasing with redshift and boosting the X-ray flux. On the contrary, in the optical, their SED is dominated either by galaxy starlight or by dust-attenuated nuclear emission or by a combination of both. For these reasons, the $k$-correction works in the opposite direction with respect to the X-ray one: moving to high redshifts, the faint rest frame optical/UV emission is shifted to the $i$ band explaining the progressively faint optical magnitudes (see a similar discussion in Brusa et al. 2010 and Comastri et al. 2003). The result is that the change in observed X-ray flux with increasing redshift is smaller than the change in optical $i$-band magnitude and thus obscured AGNs and galaxy SED sources at high redshift have higher X/O. Figure 14 shows that galaxy SED sources occupy the same region of the $\mathrm{X} / \mathrm{O}$ versus the redshift plane of obscured AGNs and extend to higher redshifts and larger X/O. This suggests that galaxy SED sources are mainly obscured AGNs at high redshift.

Unobscured sources have similarly steep spectral slopes in both the optical and X-rays ( $\alpha \sim 1)$, thus the two $k$-corrections are similar. For this reason, the spread in $\mathrm{X} / \mathrm{O}$ in the \pm 1 range (Figure 13) remains constant with redshift (Figure 14) and it is merely due to the spread in luminosity. Consequently, there is no correlation between $\mathrm{X} / \mathrm{O}$ and luminosity for unobscured AGNs, while it has been widely shown that X-ray to optical flux ratio correlates with hard X-ray luminosity for obscured AGNs (Fiore et al. 2003; Eckart et al. 2006; Brusa et al. 2010).

In Figure 15, the hard X-ray luminosity and the $\mathrm{X} / \mathrm{O}$ (computed in the hard band; Fiore et al. 2003) are plotted for the obscured AGNs and the galaxy SED sources at a luminosity $>10^{42} \mathrm{erg} \mathrm{s}^{-1}$. The sample consists of 762 sources. The correlation (with a slope of 1.2; black solid line) is quite strong over three orders of magnitude in luminosity (Spearman rank correlation coefficient $\rho=0.8$ ) and it is stable even when 


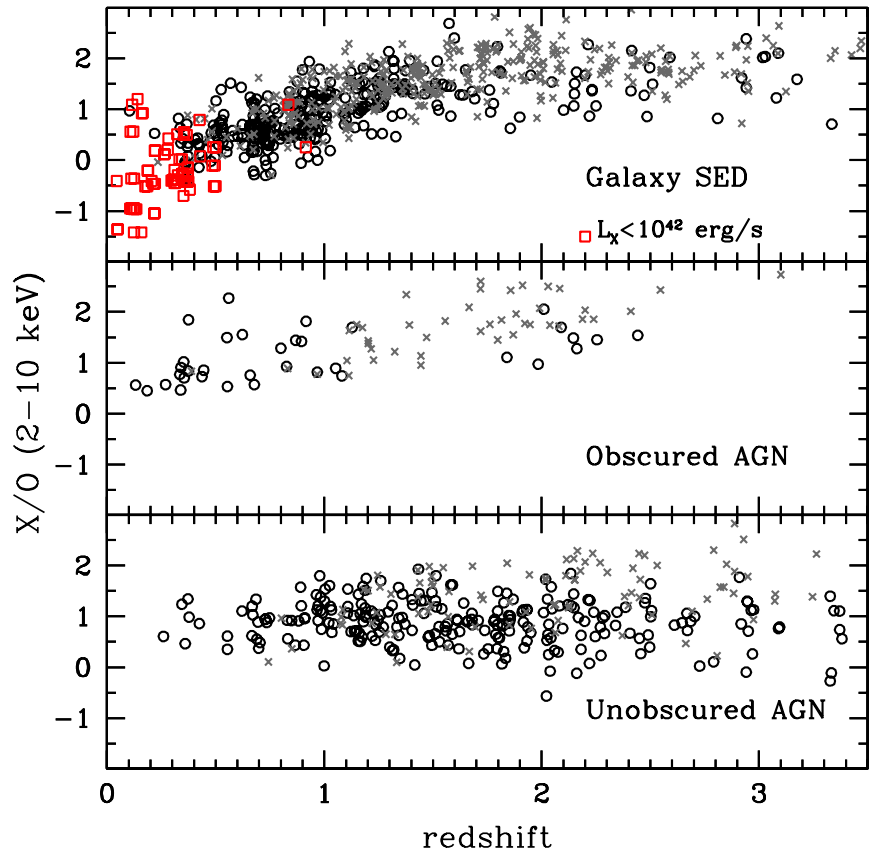

Figure 14. X-ray to optical flux ratio in the hard band vs. the redshift, dividing the sources on the basis of the optical best-fitting template. Black circles represent sources with a spectroscopic identification, gray crosses those without. The red squares represent sources with $L_{F}<10^{42} \mathrm{erg} \mathrm{s}^{-1}$.

(A color version of this figure is available in the online journal.)

the sample is divided between sources with spectroscopic and photometric redshifts. This correlation again supports the fact that there is a good coupling between the galaxy and the central $\mathrm{BH}$ over a wide range of X-ray luminosities. In the past, it has been pointed out that at faint optical magnitudes, the two quantities do not correlate (Barger et al. 2005; Civano et al. 2005 ) or follow a different relation. Brusa et al. (2010) found the same behavior for the faint $(R>23)$ XMM-COSMOS sources. We considered here the subsamples of sources at $i>23$ (450 sources) and $i>25$ (blue symbols in Figure 15). We find that the first group follows approximately the same correlation of the total sample while at fainter magnitudes there is still a correlation but with different normalization and slope (0.9; dashed line). However, a larger sample of faint sources is necessary to verify if the correlation holds, suggesting a different population of sources, or it is mainly a spread toward high $\mathrm{X} / \mathrm{O}$ due to the detection of low-luminosity host galaxies at higher redshifts. Thus, this correlation can still be used to estimate redshifts for optically bright sources, as suggested in the past (Fiore et al. 2003), but some caveats have to be considered for faint sources.

\subsubsection{X-Ray to Optical Flux Ratio and Morphology}

Recently, Pović et al. (2009a, 2009b) found an anti-correlation between the $\mathrm{X} / \mathrm{O}$ ratio and the concentration parameter $(C$; Abraham et al. 1994), which represents how the source light is distributed among the galaxy pixels. The $\mathrm{X} / \mathrm{O}$ ratio could be anti-correlated with the production efficiency of the $\mathrm{BH}$ (i.e., the Eddington ratio; Kelly et al. 2008; Pović et al. 2009a; Trump et al. 2011). Thus, the anti-correlation could be due to a dependence of the Eddington ratio and the galaxy mass, which strongly correlates with $C$ (Graham et al. 2001a, 2001b).

We correlated the C-COSMOS counterparts catalog with the Tasca et al. (2009) catalog which reports the morpho-

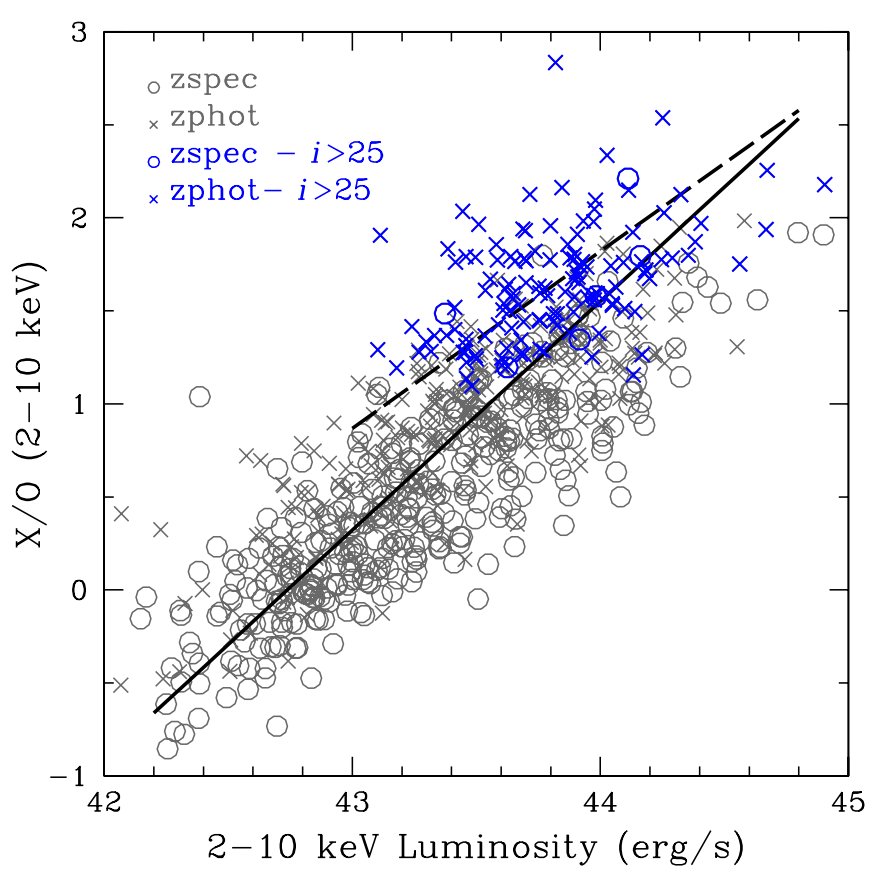

Figure 15. X-ray to optical flux ratio in the hard band vs. the hard-band $\mathrm{X}$-ray luminosity. Circles represent sources with a spectroscopic identification; crosses represent those without. The blue sources have $i>25$. The solid line is the fit to the total sample. The dashed line is the fit to the faint optical sources. (A color version of this figure is available in the online journal.)

logical parametric quantities, computed using Morpheus 2005 (Abraham et al. 2007) on HST ACS images, for several thousand COSMOS sources to $i=22.5$. In order not to be contaminated by unobscured AGNs, for which the presence of a bright nuclear source can affect the determination of the concentration index (Gabor et al. 2009), we excluded from the sample sources classified as point-like from the ACS catalog (Leauthaud et al. 2007) and also sources classified as unobscured based on their SED. Also, to avoid the contamination by normal star-forming galaxies we excluded from the sample sources with luminosity in the full band $<10^{42} \mathrm{erg} \mathrm{s}^{-1}$. In Figure 16, the X/O computed in the hard band and $\log (C)$ are plotted for the 561 sources with the above selection.

We find a significant anti-correlation (Spearman coefficient $\rho=0.45$; solid line in Figure 16) between the two quantities. The scatter around the correlation is large; however, the significance is the same as that of Povic et al. (2009a, 2009b). The best-fit relation is $\mathrm{X} / \mathrm{O}=-0.057-1.57 \times C$. We note that Pović et al. (2009a, 2009b) use different X-ray band and optical magnitudes to compute the $\mathrm{X} / \mathrm{O}$ ratio, so the normalization of their fit is quite different (dot-dashed line in Figure 16) and also their slope is steeper but this could be due to the limited number of sources in the Pović et al. (2009b) sample ( $\sim 100$ sources). The large range of $\mathrm{X} / \mathrm{O}$ covered implies that this anti-correlation is valid for a sample spanning a large range of redshifts and luminosities, thus being representative of the entire sample.

We used the same morphological catalog to classify the sources in three different classes: bulgy (red circles), disky/ spiral (blue squares), and irregular (cyan triangles). We find that most of the sources (50\%) are classified as disky/spiral, $40 \%$ as bulgy galaxies, and only $10 \%$ of the sources show an irregular morphology. This result is in agreement with recent findings (e.g., Gabor et al. 2009; Cisternas et al. 2011; Schawinski et al. 2011; Kocevski et al. 2012; M. Kriek et al., in preparation), suggesting that secular processes rather than major mergers 


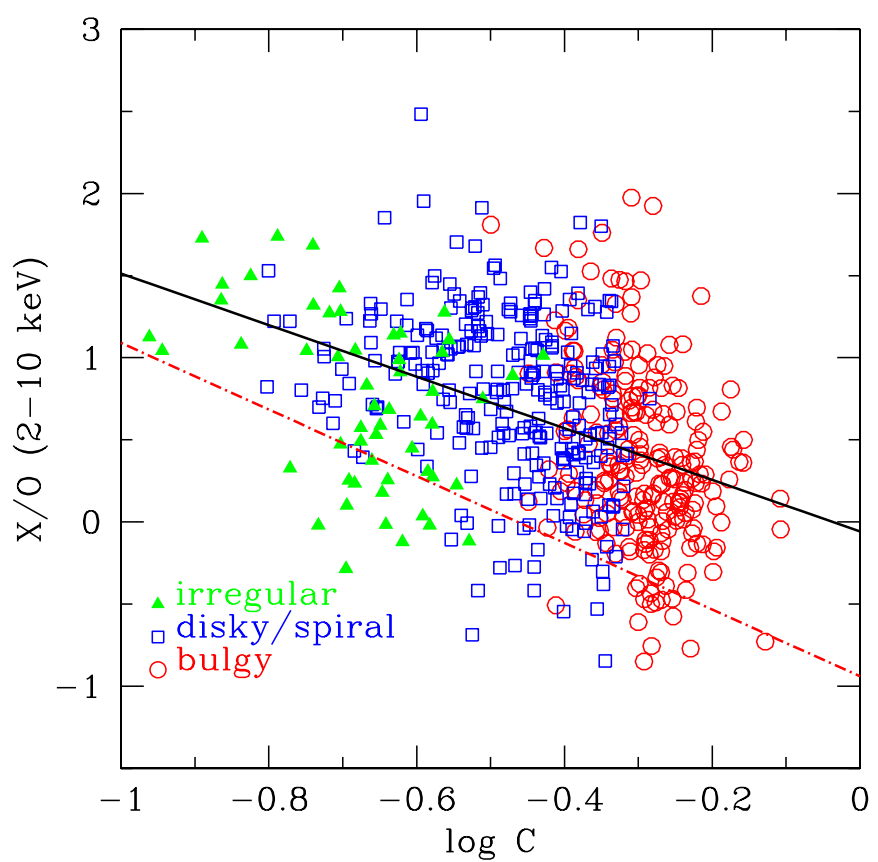

Figure 16. X-ray to optical flux ratio in the hard band vs. the concentration parameter measured in the HST ACS images. Red circles represent sources with a bulgy morphology, blue squares sources with a disky/spiral morphology, green triangles sources with an irregular morphology. The black solid line is the linear fit. The red dot-dashed line is the Pović et al. (2009b) fit.

(A color version of this figure is available in the online journal.)

govern a significant fraction of the obscured $\mathrm{BH}$ growth in the luminosity regime sampled by $\mathrm{X}$-ray surveys.

We note that the median $\mathrm{X} / \mathrm{O}$ value for the three classes increases with decreasing concentration and that the fraction of sources at $L_{X}>10^{43.5} \mathrm{erg} \mathrm{s}^{-1}$ is larger in irregular sources $(45 \%)$ than in disky/spiral $(36 \%)$ and bulgy $(25 \%)$ galaxies. Thus, sources with irregular morphologies tend to have higher $\mathrm{X}$-ray luminosities $\left(>10^{43.5} \mathrm{erg} \mathrm{cm}^{-2} \mathrm{~s}^{-1}\right.$ in the hard band) and higher $\mathrm{X} / \mathrm{O}$ (indicating higher obscuration) than sources with undisturbed (spiral or bulgy) morphologies. This observed behavior is currently favored by models of merger-driven AGN fueling (e.g., Hopkins et al. 2008), where the obscured accretion phase should happen in sources with an irregular morphology due to major mergers, suggesting that these models are valid in the high-luminosity regime only.

\subsection{Optical to X-Ray Color Diagrams}

To further study the nature of the sources dominated by the host galaxy light in the optical (galaxy SED sources and obscured AGNs), the X-ray properties were analyzed and compared with the optical properties.

The hardness ratio (HR) provides a first, approximate indication of the shape of the X-ray spectra. HR is defined as $\mathrm{HR}=(H-S) /(H+S)$, where $H$ is the number of counts in the hard band and $S$ is the number of counts in the soft band. Comparing the column densities derived from the spectral analysis of the brightest C-COSMOS sources (Paper IV) with their HRs, we estimated that $\mathrm{HR}=-0.2$ is an appropriate value to roughly separate sources with significant obscuration $\left(N_{\mathrm{H}}>10^{22} \mathrm{~cm}^{-2}\right)$ from effectively unobscured sources. This value is consistent with what has been usually used in the literature in other Chandra surveys (e.g., Wilkes et al. 2009), even if we have to keep in mind that it is only an approximation,

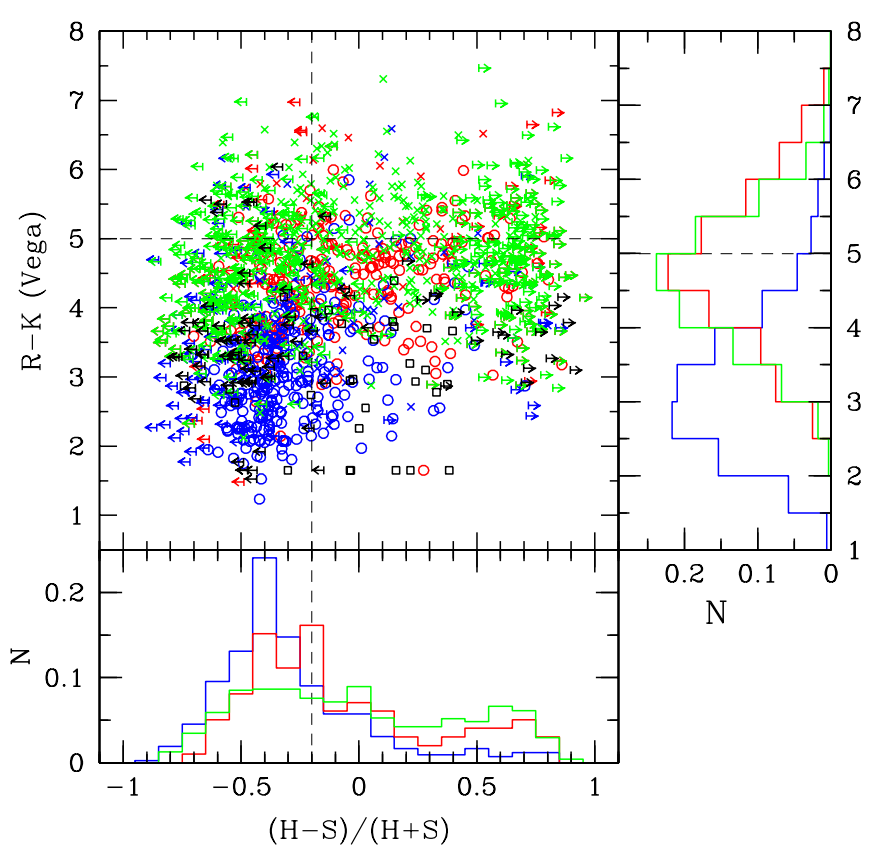

Figure 17. $R-K$ color in the Vega system vs. the X-ray hardness ratio. Circles represent sources with a spectroscopic identification, crosses those without. Arrow symbols represent sources without a detection in the soft or hard band for which a limit has been estimated. Blue: unobscured AGNs; red: obscured AGNs; green: galaxy SED sources. The black squares and arrows represent sources with $L_{F}<10^{42} \mathrm{erg} \mathrm{s}^{-1}$. In the right and bottom histograms, the distributions of the two quantities in photometric classes are plotted. The HR distributions take into account also upper limits.

(A color version of this figure is available in the online journal.)

given that the relation between X-ray column density and HR is strongly redshift-dependent (see Civano et al. 2011, Figure 1) and the statistics for faint sources are poor. For the sources not detected in the hard or soft band we computed upper or lower limits on the HR, using for each band the smallest number of counts detected in the field (3.7 in the soft band and 4.5 in the hard band).

In the optical and infrared bands, the $R-K$ color has been used in the past to select obscured sources in X-ray surveys (Brusa et al. 2005) and it has been shown that a correlation exists between $\mathrm{X} / \mathrm{O}$ and $R-K$ (Brusa et al. 2010; Rovilos et al. 2011). In Figure 17 (left panel), the $R-K$ color (computed using aperture photometry) is plotted versus the HR for all the sources, together with the distributions of the two quantities, dividing the sources in the three photometric types. The sources with X-ray luminosity $<10^{42} \mathrm{erg} \mathrm{s}^{-1}$ (red squares) are scattered across the plane without showing a correlation between HR and optical colors. These sources have been excluded from the histograms in Figure 17 (left panel).

Unobscured AGNs (blue histogram) have on average blue color $(\langle R-K\rangle=3.27)$ in the optical and soft spectra in the $\mathrm{X}$-ray $(\langle\mathrm{HR}\rangle=-0.29$, including upper limits), even if a tail at higher $\mathrm{HR}$ and $R-K$ is present, in agreement with other findings of red quasars with broad lines (Glikman et al. 2007; Urrutia et al. 2008; Young et al. 2008). The sources fitted with a galaxy template have $\mathrm{HR}$ and $R-K$ distributions consistent with those of obscured AGNs, with $\langle\mathrm{HR}\rangle=-0.01$ and -0.08 , and $\langle R-K\rangle=4.65$ and 4.83 , respectively. Similar numbers are obtained when upper limits are not considered. If BLAGNs and non-BLAGNs are considered, the HR and $R-K$ colors are consistent with those found if the photometric classification is used $(\langle\mathrm{HR}\rangle=-0.31$ and -0.04 , and $\langle\mathrm{HR}\rangle=3.1$ and 4.3$)$. 


\section{SUMMARY}

In this paper we have presented the identification procedure, the catalog, and some basic properties of the X-ray point-like sources detected in the C-COSMOS project. We summarize the most important results below.

1. The procedure for the association of the counterparts, performed via the likelihood ratio technique in three different bands $(i, K, 3.6 \mu \mathrm{m})$, has produced very high identification rates $(97 \%)$, after matching the results in the three bands and also thanks to the good correlation of near-infrared data with X-ray data at the depth of C-COSMOS.

2. Optical and near-infrared information is available for all the counterparts in the sample except for 10 objects, two of which are truly empty fields.

3. Secure spectroscopic redshifts with two or more emission and/or absorption lines are available for 906 sources. Of them $35 \%$ are identified as BLAGNs and $59 \%$ do not show broad lines but only narrow lines or absorption lines. The remaining sources are spectroscopically identified stars.

4. Photometric redshifts are available for 1693 sources. According to the photometric classification, based on the fitting of the SED, most of the sources are best fitted by a pure galaxy template $(65 \%)$, though in the whole sample only $\sim 10 \%$ of the sources have luminosity $<10^{42} \mathrm{erg} \mathrm{s}^{-1}$ possibly due to non-nuclear emission.

5. C-COSMOS occupies a sweet spot in the X-ray survey "wedding cake": the perfect match between the X-ray and optical/near-infrared depth allows us to classify the $z=0-5$ $\mathrm{X}$-ray sources using just their photometric data; the large area provides sizable samples of rare sources (e.g., high- $z$ sources) and allows us to recognize a very large sample of obscured sources $(71 \%$ on the basis of the photometric SED fitting and the X-ray luminosity), either best fitted in the optical by an obscured (hybrid) AGN template or by a galaxy template. These obscured sources start to dominate the whole X-ray population below fluxes of 4 $\times 10^{-15} \mathrm{erg} \mathrm{cm}^{-2} \mathrm{~s}^{-1}(0.5-2 \mathrm{keV})$. The optical and X-ray properties (redshifts, $\mathrm{X} / \mathrm{O}, \mathrm{HR}, R-K$, and $N_{\mathrm{H}}$ ) confirm that most of them are highly obscured $z=1-2$ AGNs. The X-ray band remains the only band able to isolate this population of obscured sources which show a normal galaxy SED in the optical.

6. Thanks to the large sample of AGNs in the C-COSMOS survey, we revised-both in the soft and in the hard band-the X-ray to optical flux ratio locus, originally defined in the soft band. We combined the C-COSMOS and XMM-COSMOS catalogs, providing a sample of 2214 sources in the soft band and 1676 in the hard band, spanning two orders of magnitude in X-ray flux. Ninety percent of the sources lie in a region slightly offset from the "classic locus" in the soft band, while in the hard band the new locus is offset by $\Delta(\mathrm{X} / \mathrm{O}) \sim 0.5$ with respect to the "hard-band locus." We also provide the best fits to the X-ray flux and total $i$-band magnitude for the AGN sample in both bands.

7. We also presented the X-ray to $K$ and $3.6 \mu \mathrm{m}$ flux ratios which shows a smaller spread of the sources, indicating a strong correlation of X-rays with near-infrared bands for both obscured and unobscured AGNs, as already pointed out in the identification procedure.

8. The $\mathrm{X} / \mathrm{O}$ ratio correlates with several optical, infrared, and $\mathrm{X}$-ray properties of $\mathrm{X}$-ray sources. We confirm the presence of a correlation in the hard band between the $\mathrm{X} / \mathrm{O}$ ratio and the X-ray luminosity for obscured and galaxy SED sources, as found by several authors, confirming the tight correlation between the galaxy and the $\mathrm{BH}$ properties over a wide $\mathrm{X}$-ray luminosity range and to a magnitude limit of $i=25$. This correlation has been used in the past to estimate the $\mathrm{X}$-ray luminosity and thus the redshift of X-ray sources, just using the observed fluxes. While the fit to the bright sample has a slope consistent with previous work, the fit to the faint sample $(i>25)$ has a flatter slope and different normalization, thus putting a limit on the validity of this relation as a good estimator of redshift and luminosity at faint magnitudes.

9. An anti-correlation between the $\mathrm{X} / \mathrm{O}$ in the hard band and the concentration parameter has been found, confirming the results of Pović et al. (2009a, 2009b), by using a six times larger sample. We also find that most of the high $\mathrm{X} / \mathrm{O}$ sources, thus obscured, are classified as disky/ spiral galaxies suggesting that secular processes govern a significant fraction of the obscured $\mathrm{BH}$ growth, on a wide range of X-ray luminosities $\left(10^{43}-10^{44.5} \mathrm{erg} \mathrm{s}^{-1}\right)$ and redshift. The only sources with an irregular morphology are those at the brightest luminosities, in agreement with the predictions of merger-driven fueling scenarios. The next step will be to compute galaxy masses, by using a careful SED fitting which takes into account the nuclear component in the optical band as in Lusso et al. (2011), and to confirm the correlation between $\mathrm{X} / \mathrm{O}$ and the galaxy mass.

We began the Chandra COSMOS project in the belief that it represented a sweet spot in the inevitable depth versus area tradeoff, and the work presented here validates this approach. Other C-COSMOS results (e.g., Capak et al. 2011) have shown that this survey is also powerful at identifying large-scale structures at $z>4$ spanning a large area of the sky $\left(>15^{\prime \prime}\right)$. Given that there is twice as much area in the full COSMOS field with identical and increasing multiwavelength coverage, we feel that the best path for future deep/wide extragalactic Chandra surveys would be to complete the COSMOS field $\left(2 \mathrm{deg}^{2}\right)$ to at least the present depth.

The authors thank the referee for the useful suggestions which helped to improve the paper. F.C. thanks R. D'Abrusco, A. Goulding, M. Mignoli, and M. Povic for inspiring discussions and useful comments. This work was supported in part by NASA Chandra grant number GO7-8136A (M.E., F.C., H.H.), the Blancheflor Boncompagni Ludovisi foundation (F.C.), and the Smithsonian Scholarly Studies (F.C.). In Italy this work is supported by ASI/INAF contracts I/009/10/0 and I/088/ 06 and by PRIN INAF 2010 "From the dawn of galaxy formation to the peak of the mass assembly." M.S. acknowledges support by the German Deutsche Forschungsgemeinschaft, DFG Leibniz Prize (FKZ HA 1850/28-1). In Mexico, this work has been supported by CONACyT Grant 83564 and UNAM/ DGAPA PAPIIT Grant IN110209. This work is also based on observations made with ESO Telescopes at the La Silla or Paranal Observatories under program ID 175.A-0839.

\section{REFERENCES}

Abraham, R. G., Nair, P., McCarthy, P. J., et al. 2007, ApJ, 669, 184 Abraham, R. G., Valdes, F., Yee, H. K. C., \& van den Bergh, S. 1994, ApJ, 432, 75

Aird, J., Nandra, K., Laird, E. S., et al. 2010, MNRAS, 401, 2531

Akiyama, M., Ohta, K., Yamada, T., et al. 2000, ApJ, 532, 700 
Alexander, D. M., Brandt, W. N., Hornschemeier, A. E., et al. 2001, AJ, 122 2156

Allevato, V., Finoguenov, A., Cappelluti, N., et al. 2011, ApJ, 736, 99

Arnouts, S., Cristiani, S., Moscardini, L., et al. 1999, MNRAS, 310, 540

Barger, A. J., Cowie, L. L., Mushotzky, R. F., et al. 2005, AJ, 129, 578

Barro, G., Pérez-González, P. G., Gallego, J., et al. 2011, ApJS, 193, 30

Bertin, E., \& Arnouts, S. 1996, A\&AS, 117, 393

Bongiorno, A., Mignoli, M., Zamorani, G., et al. 2010, A\&A, 510, A56

Bongiorno, A., Zamorani, G., Gavignaud, I., et al. 2007, A\&A, 472, 443

Brammer, G. B., van Dokkum, P. G., Franx, M., et al. 2012, ApJS, 200, 13

Brand, K., Brown, M. J. I., Dey, A., et al. 2006, ApJ, 641, 140

Brandt, W. N., \& Hasinger, G. 2005, ARA\&A, 43, 827

Brusa, M., Civano, F., Comastri, A., et al. 2010, ApJ, 716, 348

Brusa, M., Comastri, A., Daddi, E., et al. 2005, A\&A, 432, 69

Brusa, M., Comastri, A., Gilli, R., et al. 2009, ApJ, 693, 8

Brusa, M., Comastri, A., Mignoli, M., et al. 2003, A\&A, 409, 65

Brusa, M., Zamorani, G., Comastri, A., et al. 2007, ApJS, 172, 353

Capak, P., Aussel, H., Ajiki, M., et al. 2007, ApJS, 172, 99

Capak, P. L., Riechers, D., Scoville, N. Z., et al. 2011, Nature, 470, 233

Cappelluti, N., Brusa, M., Hasinger, G., et al. 2009, A\&A, 497, 635

Cardamone, C. N., Urry, C. M., Damen, M., et al. 2008, ApJ, 680, 130

Ciliegi, P., Zamorani, G., Bondi, M., et al. 2005, A\&A, 441, 879

Cimatti, A., Daddi, E., \& Renzini, A. 2006, A\&A, 453, L29

Cisternas, M., Jahnke, K., Inskip, K.J., et al. 2011, ApJ, 726, 57

Civano, F., Brusa, M., Comastri, A., et al. 2011, ApJ, 741, 91

Civano, F., Comastri, A., \& Brusa, M. 2005, MNRAS, 358, 693

Civano, F., Elvis, M., Lanzuisi, G., et al. 2010, ApJ, 717, 209

Civano, F., Elvis, M., Lanzuisi, G., et al. 2012, ApJ, 752, 49

Civano, F., Mignoli, M., Comastri, A., et al. 2007, A\&A, 476, 1223

Cocchia, F., Fiore, F., Vignali, C., et al. 2007, A\&A, 466, 31

Comastri, A., Brusa, M., Mignoli, M., \& HELLAS2XMM Team 2003, Astron. Nachr., 324, 28

Comastri, A., Mignoli, M., Ciliegi, P., et al. 2002, ApJ, 571, 771

Della Ceca, R., Maccacaro, T., Caccianiga, A., et al. 2004, A\&A, 428, 383

Donley, J. L., Koekemoer, A. M., Brusa, M., et al. 2012, ApJ, 748, 142

Donley, J. L., Rieke, G. H., Pérez-González, P. G., \& Barro, G. 2008, ApJ, 687, 111

Drake, J., Wright, N., \& Chandra Cyg Ob2 Team. 2009, in Proc. Conf. Chandra's First Decade of Discovery, ed. S. Wolk, A. Fruscione, \& D. Swartz (http://cxc.harvard.edu/ChandraDecade/Proceedings/starts_formation. html\#abs 1.3)

Eckart, M. E., Stern, D., Helfand, D. J., et al. 2006, ApJS, 165, 19

Elvis, M., Civano, F., Vignali, C., et al. 2009, ApJS, 184, 158

Elvis, M., Heng, H. H., Civano, F., et al. 2012, ApJ, submitted

Elvis, M., Wilkes, B. J., McDowell, J. C., et al. 1994, ApJS, 95, 1

Fan, X., Strauss, M. A., Richards, G. T., et al. 2006, AJ, 131, 1203

Ferrarese, L., \& Merritt, D. 2000, ApJ, 539, L9

Fiore, F., Brusa, M., Cocchia, F., et al. 2003, A\&A, 409, 79

Fiore, F., Puccetti, S., Brusa, M., et al. 2009, ApJ, 693, 447

Fiore, F., Puccetti, S., Grazian, A., et al. 2012, A\&A, 537, A16

Fotopoulou, S., Salvato, M., Hasinger, G., et al. 2012, ApJS, 198, 1

Gabor, J. M., Impey, C. D., Jahnke, K., et al. 2009, ApJ, 691, 705

Gebhardt, K., Bender, R., Bower, G., et al. 2000, ApJ, 539, L13

Gilli, R., Zamorani, G., Miyaji, T., et al. 2009, A\&A, 494, 33

Glikman, E., Helfand, D. J., White, R. L., et al. 2007, ApJ, 667, 673

Goulding, A. D., Forman, W. R., Hickox, R. C., et al. 2012, ApJS, in press (arXiv:1206.6884)

Graham, A. W., Erwin, P., Caon, N., \& Trujillo, I. 2001a, ApJ, 563, L11

Graham, A. W., Trujillo, I., \& Caon, N. 2001b, AJ, 122, 1707

Green, P. J., Aldcroft, T. L., Richards, G. T., et al. 2009, ApJ, 690, 644

Hasinger, G., Cappelluti, N., Brunner, H., et al. 2007, ApJS, 172, 29

Hasinger, G., Miyaji, T., \& Schmidt, M. 2005, A\&A, 441, 417

Hickox, R. C., Myers, A. D., Brodwin, M., et al. 2011, ApJ, 731, 117

Hopkins, P. F., Hernquist, L., Cox, T. J., \& Kereš, D. 2008, ApJS, 175, 356

Hornschemeier, A. E., Bauer, F. E., Alexander, D. M., et al. 2003, AJ, 126, 575

Hornschemeier, A. E., Brandt, W. N., Garmire, G. P., et al. 2001, ApJ, 554, 742

Ilbert, O., Arnouts, S., McCracken, H. J., et al. 2006, A\&A, 457, 841

Ilbert, O., Capak, P., Salvato, M., et al. 2009, ApJ, 690, 1236

Ilbert, O., Salvato, M., Le Floc'h, E., et al. 2010, ApJ, 709, 644

Ikeda, H., Nagao, T., Matsuoka, K., et al. 2011, ApJ, 728, L25

Kartaltepe, J. S., Sanders, D. B., Le Floc'h, E., et al. 2010, ApJ, 709, 572

Kelly, B. C., Bechtold, J., Trump, J. R., Vestergaard, M., \& Siemiginowska, A. 2008, ApJS, 176, 355

Kewley, L. J., Dopita, M. A., Sutherland, R. S., Heisler, C. A., \& Trevena, J. 2001, ApJ, 556, 121
Kocevski, D. D., Faber, S. M., Mozena, M., et al. 2012, ApJ, 744, 148

Koekemoer, A. M., Alexander, D. M., Bauer, F. E., et al. 2004, ApJ, 600, L123

Koekemoer, A. M., Aussel, H., Calzetti, D., et al. 2007, ApJS, 172, 196

Koekemoer, A. M., Fruchter, A. S., Hook, R. N., \& Hack, W. 2003, in The 2002 HST Calibration Workshop: Hubble after the Installation of the ACS and the NICMOS Cooling System, Space Telescope Science Institute, Baltimore, MD, 2002 October 17 and 18, ed. S. Arribas, A. Koekemoer, \& B. Whitmore (Baltimore, MD: Space Telescope Science Institute), 337

Kümmel, M., Walsh, J. R., Pirzkal, N., Kuntschner, H., \& Pasquali, A. 2009, PASP, 121, 59

La Franca, F., Fiore, F., Comastri, A., et al. 2005, ApJ, 635, 864

Laird, E. S., Nandra, K., Georgakakis, A., et al. 2009, ApJS, 180, 102

Leauthaud, A., Massey, R., Kneib, J.-P., et al. 2007, ApJS, 172, 219

Lehmann, I., Hasinger, G., Schmidt, M., et al. 2001, A\&A, 371, 833

Lehmer, B. D., Xue, Y. Q., Brandt, W. N., et al. 2012, ApJ, 752, 46

Lilly, S. J., Le Brun, V., Maier, C., et al. 2009, ApJS, 184, 218

Lilly, S. J., Le Fèvre, O., Renzini, A., et al. 2007, ApJS, 172, 70

Luo, B., Bauer, F. E., Brandt, W. N., et al. 2008, ApJS, 179, 19

Luo, B., Brandt, W. N., Xue, Y. Q., et al. 2010, ApJS, 187, 560

Luo, B., Brandt, W. N., Xue, Y. Q., et al. 2011, ApJ, 740, 37

Lusso, E., Comastri, A., Vignali, C., et al. 2010, A\&A, 512, A34

Lusso, E., Comastri, A., Vignali, C., et al. 2011, A\&A, 534, A110

Maccacaro, T., Gioia, I. M., Wolter, A., Zamorani, G., \& Stocke, J. T. 1988, ApJ, 326,680

Mainieri, V., Bergeron, J., Hasinger, G., et al. 2002, A\&A, 393, 425

Magorrian, J., Tremaine, S., Richstone, D., et al. 1998, AJ, 115, 2285

Mainieri, V., Hasinger, G., Cappelluti, N., et al. 2007, ApJS, 172, 368

Mainieri, V., Rosati, P., Tozzi, P., et al. 2005, A\&A, 437, 805

Mainieri, V., Vignali, C., Merloni, A., et al. 2010, A\&A, 514, A85

McCracken, H. J., Capak, P., Salvato, M., et al. 2010, ApJ, 708, 202

Merloni, A., Bongiorno, A., Bolzonella, M., et al. 2010, ApJ, 708, 137

Merloni, A., \& Heinz, S. 2008, MNRAS, 388, 1011

Mignoli, M., Pozzetti, L., Comastri, A., et al. 2004, A\&A, 418, 827

Murray, S. S., Kenter, A., Forman, W. R., et al. 2005, ApJS, 161, 1

Perola, G. C., Puccetti, S., Fiore, F., et al. 2004, A\&A, 421, 491

Pović, M., Sánchez-Portal, M., Pérez García, A. M., et al. 2009a, ApJ, 706,810

Pović, M., Sánchez-Portal, M., Pérez García, A. M., et al. 2009b, ApJ, 702, L51

Prescott, M. K. M., Impey, C. D., Cool, R. J., \& Scoville, N. Z. 2006, ApJ, 644, 100

Puccetti, S., Vignali, C., Cappelluti, N., et al. 2009, ApJS, 185, 586

Rovilos, E., Fotopoulou, S., Salvato, M., et al. 2011, A\&A, 529, A135

Salvato, M., Hasinger, G., Ilbert, O., et al. 2009, ApJ, 690, 1250

Salvato, M., Ilbert, O., Hasinger, G., et al. 2011, ApJ, 742, 61

Sanders, D. B., Salvato, M., Aussel, H., et al. 2007, ApJS, 172, 86

Schawinski, K., Treister, E., Urry, C. M., et al. 2011, ApJ, 727, L31

Schinnerer, E., Smolčić, V., Carilli, C. L., et al. 2007, ApJS, 172, 46

Schmidt, M., Hasinger, G., Gunn, J., et al. 1998, A\&A, 329, 495

Scoville, N., Aussel, H., Brusa, M., et al. 2007, ApJS, 172, 1

Silverman, J. D., Green, P. J., Barkhouse, W. A., et al. 2008, ApJ, 679, 118

Silverman, J. D., Kampczyk, P., Jahnke, K., et al. 2011, ApJ, 743, 2

Soltan, A. 1982, MNRAS, 200, 115

Steffen, A. T., Barger, A. J., Capak, P., et al. 2004, AJ, 128, 1483

Stocke, J. T., Morris, S. L., Gioia, I. M., et al. 1991, ApJS, 76, 813

Sutherland, W., \& Saunders, W. 1992, MNRAS, 259, 413

Taniguchi, Y., Scoville, N., Murayama, T., et al. 2007, ApJS, 172, 9

Tasca, L. A. M., Kneib, J.-P., Iovino, A., et al. 2009, A\&A, 503, 379

Treister, E., Urry, C. M., Chatzichristou, E., et al. 2004, ApJ, 616, 123

Trichas, M., Green, P. J., Silverman, J. D., et al. 2012, ApJS, 200, 17

Trouille, L., Barger, A. J., \& Tremonti, C. 2011, ApJ, 742, 46

Trump, J. R., Impey, C. D., Elvis, M., et al. 2009a, ApJ, 696, 1195

Trump, J. R., Impey, C. D., Kelly, B. C., et al. 2011, ApJ, 733, 60

Trump, J. R., Impey, C. D., McCarthy, P. J., et al. 2007, ApJS, 172, 383

Trump, J. R., Impey, C. D., Taniguchi, Y., et al. 2009b, ApJ, 706, 797

Urrutia, T., Lacy, M., \& Becker, R.H. 2008, ApJ, 674, 80

van Dokkum, P. G., Brammer, G., Fumagalli, M., et al. 2011, ApJ, 743, L15

Weisskopf, M. C., Brinkman, B., Canizares, C., et al. 2002, PASP, 114, 1

Wilkes, B. J., Kilgard, R., Kim, D.-W., et al. 2009, ApJS, 185, 433

Willott, C. J., Delorme, P., Omont, A., et al. 2007, AJ, 134, 2435

Wright, N. J., Drake, J. J., \& Civano, F. 2010, ApJ, 725, 480

Wuyts, S., Labbé, I., Schreiber, N. M. F., et al. 2008, ApJ, 682, 985

Xue, Y. Q., Luo, B., Brandt, W. N., et al. 2011, ApJS, 195, 10

Young, M., Elvis, M., \& Risaliti, G. 2008, ApJ, 688, 128

Zamojski, M. A., Schiminovich, D., Rich, R. M., et al. 2007, ApJS, 172, 468 